\title{
ERNEST

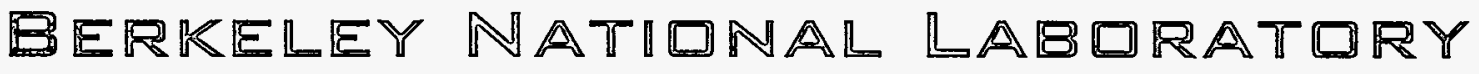

Numerical Simulation Experiments on Water Seepage Patterns in Heterogeneous, Unsaturated Rock Fractures

K. Pruess

Earth Sciences Division

May 1996 


\section{DISCLAIMER}

This document was prepared as an account of work sponsored by the United States Government. While this document is believed to contain correct information, neither the United States Government nor any agency thereof, nor The Regents of the University of Califomia, nor any of their employees, makes any warranty, express or implied, or assumes any legal responsibility for the accuracy, completeness, or usefulness of any information, apparatus, product, or process disclosed, or represents that its use would not infringe privately owned rights. Reference herein to any specific commercial product, process, or service by its trade name, trademark, manufacturer, or otherwise, does not necessarily constitute or imply its endorsement, recommendation, or favoring by the United States Government or any agency thereof, or The Regents of the University of Califomia. The views and opinions of authors expressed herein do not necessarily state or reflect those of the United States Government or any agency thereof, or The Regents of the University of California.

Available to DOE and DOE Contractors from the Office of Scientific and Technical Information

P.O. Box 62, Oak Ridge, TN 37831

Prices available from (615) $576-8401$

Available to the public from the

National Technical Information Service

U.S. Department of Commerce

5285 Port Royal Road, Springfield, VA 22161

Enest Orlando Lawrence Berkeley National Laboratory is an equal opportunity employer. 
LBL - 38883

UC\# 403

\title{
Numerical Simulation Experiments on Water Seepage Patterns in Heterogeneous, Unsaturated Rock Fractures
}

\author{
K. Pruess
}

Earth Sciences Division

Lawrence Berkeley National Laboratory

University of California

Berkeley, CA 94720

May 1996

This work was supported, in part, by the Director, Office of Civilian Radioactive Waste Management, and by the Director, Office of Energy Research, Office of Health and Environmental Sciences, Biological and Environmental Research Program, of the U.S. Department of Energy under Contract No. DE-AC03-76SF00098 
Recycled Paper 


\section{DISCLAIMER}

Portions of this document may be illegible in electronic image products. Images are produced from the best available original document. 


\title{
Numerical Simulation Experiments on Water Seepage Patterns in Heterogeneous, Unsaturated Rock Fractures
}

\author{
K. Pruess \\ Earth Sciences Division, Lawrence Berkeley National Laboratory \\ University of California, Berkeley, CA 94720
}

\begin{abstract}
Water seepage has been numerically simulated in heterogeneous fractures, which were conceptualized as two-dimensional heterogeneous porous media. Flow was found to proceed in dendritic patterns along preferential paths, giving rise to such features as localized ponding and bypassing. Limited parameter variation studies have shown strong dependence of seepage patterns on fracture permeability and applied flow rate. The temporal evolution of seeps proceeds on a vast range of time scales. This casts doubt on the applicability of steady-state concepts for water migration in thick unsaturated zones of fractured rock where infiltration is episodic. An approximate invariance of seepage behavior was derived for simultaneous space-and-time scaling. Numerical simulation experiments have confirmed this invariance, as well as its limits of applicability.
\end{abstract}

\section{Introduction}

Seepage of water in isothermal conditions in variably saturated media is usually described with Richards' equation

$$
\frac{\partial}{\partial t} \theta=\operatorname{div}[\mathrm{K} \nabla \mathrm{h}]
$$

where $t$ is time, $\theta$ is specific moisture content, $\mathrm{K}$ is hydraulic conductivity, and $\mathrm{h}$ is hydraulic head. Eq. (1) may be re-written in multiphase notation, as follows (Oldenburg and Pruess, 1993)

$$
\frac{\partial}{\partial t} \phi S_{1} \rho_{1}=\operatorname{div}\left[\frac{k k_{r l} \rho_{l}^{2} g}{\mu_{1}} \nabla\left(\frac{P_{1}}{\rho_{1} g}+z\right)\right]
$$


Here, $\phi$ is porosity, $S_{l}$ is liquid (water) saturation, $\rho_{1}$ is liquid density, $k$ is absolute permeability, $\mathrm{k}_{\mathrm{rl}}$ is relative permability, $\mathrm{g}$ is acceleration of gravity, $\mu_{1}$ is viscosity, $\mathrm{P}_{1}=\mathrm{P}_{\text {gas }}+\mathrm{P}_{\text {cap }}$ is liquid phase pressure, and $\mathrm{z}$ is the vertical coordinate (positive upward). Approximations implicit in Richards' equation include that the gas phase acts as a passive bystander during water flow, with negligible pressure changes, and that water density and viscosity are constant. Further implied are all the approximations that are inherent in (multiphase extensions of) Darcy's law, such as absence of inertial effects, and applicability of relative permeability and capillary pressure concepts. Also implied is volume averaging on some scale, although Eqs. (1) and (2) do not spell out what the spatial scale of volume averaging is.

For heterogeneous media, the hydrologic parameters $\left(k, \phi, \mathrm{P}_{\text {cap }}, \ldots\right)$ are expected to show random spatial variation with certain correlation structures, and Eqs. $(1,2)$ are stochastic partial differential equations (PDEs). The present paper focusses on permeability heterogeneity in the fracture plane. This is believed to be the dominant control on seepage behavior, although spatial variability may also be present in the functional relationships between relative permeability, capillary pressure, and liquid saturation. The transient evolution of saturation (or, equivalently, liquid pressure) in response to certain applied boundary conditions, and sinks and sources, will reflect the heterogeneity of the underlying porosity and permeability distribution. For many applications, such as for assessment of contaminant migration and the performance of waste repositories, we are interested not in the spatial and temporal details of flow but only in flow properties averaged over certain scales. A fundamental question then arises: can such averages be derived from an average saturation field $\mathrm{S}_{1}(\mathrm{x}, \mathrm{t})$, obtained by solving some simplified form of Eq. (2) with volume-averaged coefficients, or do we need to tackle the much more difficult problem of solving Eq. (2) with detailed representation of heterogeneity, perhaps for many realizations, and then average the results? Noting that the relative permeability and capillary pressure relationships in Eq. (2) usually are highly non-linear, we expect that flow behavior and saturation distributions calculated by averaging the heterogeneous porosity and permeability fields, and spatially variable boundary and sink/source conditions, may be quite different from averages obtained for ensembles of heterogeneous fields. It has also been shown that volume averaging will in general not only give rise to effective "upscaled" parameters, but may give rise to the emergence of novel "effective processes" which do not necessarily have a small-scale counterpart (Pruess, 1994, 1995). Accordingly it appears that averaging the inputs into Eq. (2) will, in general, not be a viable approach for obtaining averages for the outputs.

The conventional approach to describing the seepage of (aqueous or non-aqueous) liquids in partially saturated fractured media employs macroscale continuum concepts (Peters and 
Klavetter, 1988). Large-scale volume averaging is used for homogenizing heterogeneous fracture and matrix permeabilities, and to average out spatially variable infiltration rates applied at the system boundary, e.g., at the land surface, or at lithologic contacts between porous and fractured units. On the basis of these volume-averaged concepts, water migration along sub-vertical fractures is then typically predicted as proceeding in the form of smooth sheets, and being subject to strong imbibition effects into the partially saturated rock matrix (Nitao and Buscheck, 1991; Wang and Narasimhan, 1993; Eaton et al., 1996).

There is mounting evidence for non-volume-averaged behavior from field sites with thick unsaturated zones. At Yucca Mountain, Nevada, elevated levels of bomb pulse ${ }^{36} \mathrm{Cl}$ have been found at several hundred meters depth, indicating that water was able to migrate from the land surface to these horizons over time periods not exceeding a few decades (Liu et al., 1995). At nearby Rainier Mesa, persistent highly localized flow of water from fractures into drifts was observed at depths of several hundred meters beneath the land surface (Lawrence Berkeley Laboratory, 1991; Wang et al., 1993). Typically, only portions of fractures carried water, and the chemical composition of water obtained from fractures was substantially different from that of water samples extracted from the nearby rock matrix. At the Superior mine in Arizona, water inflow from fractures into mine drifts at several hundred meter depth varied with precipitation at the surface, with a lag time of only a few months (D. Chesnut, private communication, 1996). At a field site in the Negev desert, Israel, man-made tracers were observed to rapidly migrate across a thick unsaturated zone of fractured chalk (Nativ et al., 1995). All these observations indicate that water is able to migrate downward rather rapidly along localized preferential flow paths through fracture networks in partially saturated rocks, without being imbibed into the rock matrix. Localized preferential flow of water along non-horizontal fractures has also been observed in laboratory experiments, and has been modeled using percolation theory concepts (Nicholl et al., 1993; Glass, 1993; Nicholl et al., 1994; Geller and Pruess, 1995).

Recognizing that fast water flow along localized preferential paths may play an important role in unsaturated fractured rock, several researchers have suggested to abandon continuum-type treatments based on PDEs such as Eq. (1), and have proposed to instead conceptualize unsaturated flow in heterogeneous fractured media as a stochastic distributions of localized seeps (Gauthier et al., 1992; Gauthier, 1994; Chesnut, 1992, 1994). In the cited references, distributions of seeps were introduced in an ad hoc fashion. The objective of the present work is to propose and explore a continuum-based mechanistic model for localized seeps and their properties. Fractures are conceptualized as two-dimensional heterogeneous porous media (Pruess et al., 1990a; Pruess and Tsang, 1990). We assume that Richards' equation and its implied approximations, such as 
applicability of relative permeability and capillary pressure concepts, are valid for such media on a scale of $0.1-1 \mathrm{~m}$. High-resolution numerical simulations of unsaturated flow are then performed in which we explicitly consider fracture heterogeneity on a sub-meter scale. The TOUGH2 code was used for the simulations, augmented with a set of preconditioned conjugate gradient solvers (Moridis and Pruess, 1995) and a special fluid property module 'EOS9' for Richards' equation (Pruess, 1994).

\section{Heterogeneous Fractures}

Natural rock fractures are expected to encompass a vast variety of aperture distributions. The present work focuses on "small" fractures in hard rocks, such as tuffs, basalts, granites, or graywackes. Aspects of fracture aperture distribution that are believed to be essential for replicating natural features include (a) the presence of asperity contacts, where the fracture walls touch, (b) a more or less gradual change towards larger apertures away from the asperities, (c) small-scale fracture wall roughness, and (d) finite-size spatial correlation length among apertures (Wang and Narasimhan, 1985; Pruess and Antunez, 1995).

A synthetic heterogeneous permeability field that can represent a fracture is obtained by means of the turning bands method, as implemented by Tompson (1989). This method requires a log-normal distribution of coefficients, given by

$$
f(\zeta)=\frac{1}{\zeta \sigma \sqrt{2 \pi}} \exp \left(-\frac{(\ln (\zeta)-<\ln (\zeta)>)^{2}}{2 \sigma^{2}}\right)
$$

The particular lognormal distribution shown in Fig. 1 corresponds to parameters of $<\ln (\zeta)>=1$, standard deviation $\sigma=1.5$. Using $10,000 \zeta$-coefficients randomly picked from the distribution Eq. (3), a two-dimensional rectangular grid of $100 \times 100$ coefficients $\zeta_{\mathrm{ij}}$ is then generated as a spatiallycorrelated stochastic field, with spatial correlation lengths of 1 grid unit in the horizontal direction, 0.5 in the vertical. The log-normal distribution has zero probability for the value $\zeta=0$, hence cannot represent asperity contacts. To achieve the desired asperity contacts (regions of zero permeability), the log-normally distributed coefficients $\zeta_{\mathrm{ij}}$ are "shifted" according to

$$
\zeta_{\mathrm{ij}} \rightarrow \zeta_{\mathrm{ij}}{ }^{\prime}=\max \left(\zeta_{\mathrm{ij}}-\Delta, 0\right)
$$

The $\zeta_{\mathrm{ij}}{ }^{\prime}$ coefficients are then attached to a finite difference grid of $100 \times 100=10,000$ square blocks of $0.2 \mathrm{~m}$ length, each of which is assigned a permeability $\mathrm{k}_{\mathrm{ij}}=\zeta_{\mathrm{ij}}{ }^{\prime} \times \mathrm{k}_{\mathrm{ref}}$, where reference 
permeability is typically chosen as $\mathrm{k}_{\mathrm{ref}}=10^{-9} \mathrm{~m}^{2}$. The capillary pressure functions are scaled along with permeability on a grid-block-by-grid-block basis according to $P_{\text {cap }} \rightarrow P_{c a p}{ }^{\prime}=P_{c a p} / V\left(\zeta_{i j}{ }^{\prime}\right)$ (Leverett, 1941).

For the flow simulations reported here the shift $\Delta$ was chosen as 1 , which resulted in a fraction of $26.4 \%$ of the permeability modifiers $\zeta_{\mathrm{ij}}$ ' being equal to 0 , corresponding to asperity contacts (Fig. 2). This fraction of fracture wall contact area is considered reasonable, based on analyses of fracture wall coatings from Yucca Mountain (Wang and Narasimhan, 1993). For a reference permeability of $10^{-9} \mathrm{~m}^{2}$, the corresponding parallel-plate hydraulic aperture (Witherspoon et al., 1980) is $b=\sqrt{ }(12 \mathrm{k})=.1095 \mathrm{~mm}$. It is well known that in natural roughwalled rock fractures the "volumetric aperture" (defined as fracture void volume per unit surface area) is considerably larger than the hydraulic aperture, often by orders of magnitude (Abelin et al., 1987). This is explained by the fact that fracture permeability is controlled by the smallest apertures (largest flow resistance), while fracture void volumes are dominated by the largest apertures. We do not use a parallel-plate concept, but instead model the fracture as a two-dimensional heterogeneous porous medium. Seepage behavior is determined, generally speaking, by an interplay between permeability heterogeneity, capillary pressure and relative permeability effects, and spatial variations in volumetric aperture. Permeability heterogeneity and associated variations in strength of capillary pressures are expected to be the dominant control on seepage behavior. Accordingly, we neglect possible spatial variations in fracture volumetric aperture in this study, except that in regions of zero permeability apertures were assumed to be zero. The fracture is represented as a $10 \mathrm{~mm}$ thick domain of "large" permeability, sandwiched between tight matrix rock. Intrinsic porosity of the fracture is chosen as $\phi=0.35$, corresponding to a volumetric aperture of $3.5 \mathrm{~mm}$. For a reference permeability of $\mathrm{k}_{\mathrm{ref}}=10^{-9} \mathrm{~m}^{2}$, the permeability-thickness product of the fracture is $10^{-11} \mathrm{~m}^{3}$, or 10 darcy-meters. Analysis of barometric pressure propagation suggests that such values are reasonable for fractures in welded tuffs at Yucca Mountain, (R. Ahlers, private communication, 1996).

Matrix permeability of unfractured welded tuffs is typically of order $10^{-18} \mathrm{~m}^{2}$ (1 microdarcy) or lower. This is very small in comparison to typical permeabilities of tens or hundreds of darcies $\left(10^{-9}-10^{-7} \mathrm{~m}^{2}\right)$ in the fracture plane. Thus, rock matrix permeability will have little impact on seepage behavior over shorter time periods (days), although water imbibition into the rock matrix may be an important long-term effect. This paper is mainly concerned with flow of injected water in the fracture plane over relatively short time periods (days); accordingly, matrix permeability was neglected. 


\section{Flow Simulations}

The flow simulations were carried out by injecting water at various time-independent rates at the top of the fracture shown in Fig. 2. Injection was made over different $1 \mathrm{~m}$ intervals, corresponding to five neighboring grid blocks, centered at distances of, respectively, 6.5, 8.5, 10.5 , and $12.5 \mathrm{~m}$ from the left boundary of the fracture. Injection at a prescribed rate into a heterogeneous medium raises some non-trivial issues. In our case it would not be acceptable to simply distribute the desired injection rate uniformly over the grid blocks adjacent to the injection boundary. Indeed, because of the strong heterogeneities present some of the injection blocks may be unable to take a $1 / 5$ share of the total injection rate without large and unphysical pressure excursions. Wè accomplish injection by attaching an additional injection grid block of $1 \mathrm{~m}$ horizontal length and "average" permeability (see below) to the top of the fracture at the desired injection interval. As water is injected at a prescribed rate into this block, water saturation will increase and outflow into the fracture blocks beneath the injection block will take place. The outflow will in general partition non-uniformly among the fracture blocks. After steady flow conditions are reached near the injection interval, the injection block will remain in conditions of constant water saturation and capillary pressure. Thus, after a rapid initial transient, the boundary conditions at the top of the fracture will be constant capillary pressure over the injection interval, even though the boundary condition specified for the injection block is "constant rate."

Lateral boundaries were "no flow," and at the bottom a unit head gradient boundary condition was imposed. This is the condition that would develop, on average, if we had specified the fracture to be of much larger vertical length, situated "far" above the water table (beyond the capillary fringe). In other words, the bottom unit head gradient boundary condition enables us to model flow in a fracture of finite vertical extent, without unphysical finite-size effects propagating upwards from the bottom boundary. The unit head gradient condition was enforced by effectively giving the bottom row of grid blocks infinite volume, and turning off the capillary pressure gradient term when computing flow into these blocks. Recent theoretical and experimental work suggests that relative permeability and capillary pressure behavior of fractures is similar to that of highly permeable media with intergranular porosity (Pruess and Tsang, 1990; Firoozabadi and Hauge, 1990; Persoff and Pruess, 1995). Accordingly we used the customary van Genuchten correlations, with parameters chosen as for coarse sands (see Figs. 3, 4; van Genuchten, 1980; Pruess, 1996). Initial water saturation in the fracture was specified at the irreducible level of $S_{1}=$ $S_{l r}=0.15$, so that all additional water introduced into the fracture would be mobile. A smaller value for the parameter $S_{\mathrm{Ir}}$ was specified in the capillary pressure as compared to the relative permeability function, to avoid the unphysical behavior of $P_{\text {cap }} \rightarrow-\infty$ as $k_{r l} \rightarrow 0$. A summary of problem specifications appears in Table 1. 
Table 1. Parameters for heterogeneous fracture flow simulations.

\begin{tabular}{|c|c|}
\hline $\begin{array}{l}\text { Reference permeability } \\
\text { Porosity }\end{array}$ & $\begin{array}{l}\mathrm{k}_{\mathrm{ref}}=10^{-9} \mathrm{~m}^{2} \\
\phi=0.35\end{array}$ \\
\hline \multicolumn{2}{|l|}{ Relative Permeability } \\
\hline $\begin{aligned} & \operatorname{van} \text { Genuchten function (1980) } \sqrt{\mathrm{s}^{*}}\left\{1-\left(1-\left[\mathrm{s}^{*}\right]^{1 / \lambda}\right)^{\lambda}\right\}^{2} \\
& \text { irreducible water saturation } \\
& \text { exponent }\end{aligned}$ & $\begin{array}{l}S^{*}=\left(S_{1}-S_{\mathrm{lr}}\right) /\left(1-S_{\mathrm{lr}}\right) \\
S_{\mathrm{lr}}=0.15 \\
\lambda=0.457\end{array}$ \\
\hline \multicolumn{2}{|l|}{ Capillary pressure } \\
\hline $\begin{array}{l}\text { van Genuchten function (1980) } \\
P_{\text {cap }}=-\left(\rho_{\mathrm{w}} \mathrm{g} / \mathrm{a}\right)\left(\left[\mathrm{S}^{*}\right]^{-1 / \lambda}-1\right) 1-\lambda \\
\text { irreducible water saturation } \\
\text { exponent } \\
\text { strength coefficient }\end{array}$ & $\begin{array}{l}\mathrm{S}^{*}=\left(\mathrm{S}_{\mathrm{l}}-\mathrm{S}_{\mathrm{lr}}\right) /\left(1-\mathrm{S}_{\mathrm{lr}}\right) \\
\mathrm{S}_{\mathrm{lr}}=0.0 \\
\lambda=0.457 \\
\mathrm{a}=50 \mathrm{~m}^{-1}\end{array}$ \\
\hline Initial Water Saturation & $S_{1}=0.15$ \\
\hline $\begin{array}{l}\text { Temperature } \\
\text { Water density } \\
\text { Water viscosity }\end{array}$ & $\begin{array}{l}20^{\circ} \mathrm{C} \\
998.3 \mathrm{~kg} / \mathrm{m}^{3} \\
1.00 \times 10^{-3} \mathrm{~Pa} . \mathrm{s}\end{array}$ \\
\hline
\end{tabular}

For comparison, we also simulated injection into a homogeneous fracture, with absolute horizontal and vertical permeabilities equal to that of the heterogeneous fractures. The overall average permeability of the (100x100 block) heterogeneous fracture was "measured" by numerical simulation, as follows. Two rows of grid blocks were attached to the top and bottom, respectively, of the heterogeneous fracture. Fully saturated conditions with constant-pressure boundaries were then specified in these rows, and a flow simulation was run to steady state. Vertical permeability was calculated from the steady flow rate and applied pressure drop, using Darcy's law. An analogous procedure was applied to obtain horizontal permeability, attaching two columns of grid blocks to the left and right edges of the heterogeneous fracture. Values of $\mathrm{k}_{\mathrm{h}}=.69 \times \mathrm{k}_{\mathrm{ref}}, \mathrm{k}_{\mathrm{v}}=.36$ $x k_{\text {ref }}$ were obtained for the horizontal and vertical permeabilities, respectively, which were then used in the homogeneous fracture simulations.

\section{Results and Discussion}

Results of our simulations are presented in Figs. 5 - 14. To provide a comparison case for the heterogeneous fracture we first present results for the homogeneous medium (Fig. 5). This is 
seen to produce a featureless seep in which water migrates straight downward under gravity, with lateral broadening due to capillary effects. Breakthrough at the bottom boundary (depth of $-19.5 \mathrm{~m}$ ) occurs after 23.81 hours, but the seep continues to broaden on a very slow time scale. The evolution following breakthrough is a very slow process, and becomes ever slower as time progresses. Our simulations show that noticeable differences to steady state remain even after $10^{5}$ years.

Figure 6 shows simulated seeps in the heterogeneous fracture for a reference injection rate of $10^{-3} \mathrm{~kg} / \mathrm{s}$, reference permeability of $10^{-9} \mathrm{~m}^{2}$, at the time of breakthrough at the bottom boundary. The seeps are quite different in appearance but share common features. Flow generally proceeds in narrow vertical or sub-vertical fingers. Several flow paths can develop from localized injection. These can either merge again or remain separate as water continues to migrate downward. Perched water bodies develop at asperity contacts which are associated with significant effects of bypassing and lateral displacements. Breakthrough times at $19.5 \mathrm{~m}$ depth vary from 14.40 to 24.19 hours, corresponding to average velocities of seep advancement from 0.81 to 1.35 $\mathrm{m} / \mathrm{hr}$ (see Table 2). Lateral displacement over the $19.5 \mathrm{~m}$ vertical migration distance varies from $-7.5 \mathrm{~m}$ to $+1.0 \mathrm{~m}$.

Table 2. Simulated results for seeps in heterogeneous fracture.

\begin{tabular}{|c|c|c|c|c|}
\hline Seep & \#1 & \# 2 & \#3 & \# 4 \\
\hline Injection point distance $(\mathrm{m})$ & 6.5 & 8.5 & 10.5 & 12.5 \\
\hline $\begin{array}{l}\text { Breakthrough time at } 19.5 \mathrm{~m} \\
\text { depth (hours) }\end{array}$ & 16.25 & 14.40 & 24.19 & 15.75 \\
\hline Average seep velocity $(\mathrm{m} / \mathrm{hr})$ & 1.20 & 1.35 & 0.81 & 1.24 \\
\hline $\begin{array}{l}\text { Lateral displacement at } \\
\text { breakthrough }(\mathrm{m})^{\dagger}\end{array}$ & -3.5 & -5.5 & -7.5 & +1.0 \\
\hline
\end{tabular}

$\dagger$ positive when displacement is to the right of the injection point, negative otherwise

There is a general tendency for the vertical advancement of seeps to slow down with time (Fig. 7). However, the behavior of seeps \# 1 and \# 2 near breakthrough shows that this is not always the case. Three of the four heterogeneous seeps advance considerably faster than the homogeneous seep. Seep \# 3 is an exception; it is the slowest to break through because it splits into two separate parts, both of which carry comparable amounts of fluid. Related to the rate of downward advance of the seeps are changes in their average width (Fig. 8). For the homogeneous 
seep, average width increases monotonically with vertical migration distance. This is generally although by no means universally true also for the heterogeneous seeps. Average liquid saturation in the homogeneous seep stabilizes after early-time transients (Fig. 9). The heterogeneous seeps go through rather significant and not necessarily monotonic changes, before settling down to average water saturations rather close to that of the homogeneous seep.

Although our sample of heterogeneous seeps is far too small to draw statistically valid conclusions, it appears that the heterogeneous seeps tend to behave quite differently than the seep calculated for a volume-averaged permeability field, advancing downward more rapidly, and having a smaller fracture wall interface area. Also, the heterogeneous seeps experience significant lateral displacement. It appears that the homogeneous seep cannot represent the average behavior of the heterogeneous seeps.

At a two orders of magnitude larger reference permeability of $10^{-7} \mathrm{~m}^{2}$ the seeps have generally smaller water saturations, because the imposed flow rate can be carried at lower relative permeability (Fig. 10). There is also less broadening of the seeps, due to the weaker capillary pressures. Both effects diminish the amount of water that needs to be injected to achieve breakthrough at the bottom. Vertical migration velocities for the four seeps are increased by factors of $2.34,1.96,2.09$, and 2.67 in comparison to the $\mathrm{k}=10^{-9} \mathrm{~m}^{2}$ case. It is seen that the speedups are quite different for the different seeps; the seep that is fastest at $\mathrm{k}=10^{-9} \mathrm{~m}^{2}$ is only the third to break through at $\mathrm{k}=10^{-7} \mathrm{~m}^{2}$. Seep \# 3 broke through at a lateral displacement of $-7.5 \mathrm{~m}$ for $\mathrm{k}=$ $10^{-9} \mathrm{~m}^{2}$, whereas at $\mathrm{k}=10^{-7} \mathrm{~m}^{2}$ lateral displacement at breakthrough is $+3.0 \mathrm{~m}$. From these comparisons it is obvious that the same underlying heterogeneity structure can produce quite different seeps at different values of reference permeability.

Capillary effects remain quite significant even at a large reference permeability of $\mathrm{k}=10^{-7}$ $\mathrm{m}^{2}$, as can be seen by arbitrarily setting $\mathrm{P}_{\text {cap }}=0$ for $\mathrm{k}=10^{-7} \mathrm{~m}^{2}$, which gives rise to much narrower fingering flow (Fig. 11). In addition overall shape and direction of the seep is affected; neglecting capillary pressure effects changes the lateral displacement at breakthrough from $+1.0 \mathrm{~m}$ to $+4.8 \mathrm{~m}$. Breakthrough time is shortened by $7.0 \%$; the surprisingly small magnitude of this effect is explained by the observation that on the different course taken for $P_{\text {cap }}=0$, the seep actually develops another ponded region (near - $18 \mathrm{~m}$ depth), which slows its vertical propagation. In general we expect that vertical advancement of seeps may be considerably faster when capillary pressures are weak. 
While breakthrough at the bottom boundary occurs in a matter of hours, the seeps continue to evolve over a hierarchy of time scales. 5.8 days after start of injection, seep \# 4 has developed a strong left branch (Fig. 12). After 115.7 days a third branch has evolved in the lower third of the domain, and considerable broadening towards the left has occurred near - $5 \mathrm{~m}$ depth. Comparison with the saturation distribution at 31.7 years indicates that additional minor saturation increases and broadening of the seep are continuing (see also Fig. 13). For a water-bearing fracture in a thick unsaturated zone, infiltration rates would be expected, depending on the depth of a particular fracture relative to the land surface, to vary in response to precipitation events on considerably shorter time scales. The slow time scales observed in our simulations suggest, then, that naturally occurring seeps should not as a rule be expected to having attained steady-state flow conditions.

Fig. 14 illustrates the rate dependence of a particular seep. As injection rates are increased by successive factors of 10 , from $10^{-4} \mathrm{~kg} / \mathrm{s}$ to $10^{-1} \mathrm{~kg} / \mathrm{s}$, water saturations generally become larger. There are also striking changes in appearance. Increasing the injection rate tends to diminish lateral fingering flow, with the $q=10^{-2} \mathrm{~kg} / \mathrm{s}$ seep most closely resembling the appearance observed for a homogeneous medium (cf. Fig. 5). When injection rate is increased beyond $10^{-2} \mathrm{~kg} / \mathrm{s}$, lateral branching again becomes stronger. This indicates that heterogeneous unsaturated media tend to behave most strongly heterogeneous at large and small water saturations, while behaving less heterogeneous at intermediate water saturations (cf. Birkhoelzer and Tsang, manuscript in preparation, 1996).

\section{Behavior under Space and Time Scaling}

In connection with waste isolation problems, an assessment of water seepage in heterogeneous fractures is often required for space and time scales far larger than what is accessible through laboratory experimentation and field observation. It is of interest, therefore, to examine the dependence of seepage behavior on the space and time scales involved (Geller and Pruess, 1995; Pruess, 1995). This can be conveniently done by considering a discretized version of Richards' equation, Eq. (2). Using integral finite differences for space and first-order finite differences for time, a space-and-time discretized version of Eq. (2) for an arbitrary reservoir subdomain (grid block) n can be written as follows (Pruess, 1991)

$$
\Delta M_{n}=\frac{\Delta t}{V_{n}} \sum_{m} A_{n m} F_{n m}+\frac{\Delta t}{V_{n}} Q_{n}
$$

Here, $\Delta M_{n}$ is the change in the accumulation term over time step $\Delta t=t^{k+1}-t^{k}$, 


$$
\Delta M_{n}=\left(\phi S_{1} \rho_{1}\right)_{n}^{k+1}-\left(\phi S_{1} \rho_{1}\right)_{n}^{k}
$$

$V_{n}$ is the volume of the grid block, and $A_{n m}$ is the interface area between grid blocks $n$ and $m$. The summation in Eq. (5) extends over all grid blocks $m$ that are connected to $n . F_{n m}$ is a finite difference approximation for the flux from grid block $m$ into $n$, given by

$$
\mathrm{F}_{\mathrm{nm}}=\mathrm{k}_{\mathrm{nm}}\left(\frac{\mathrm{k}_{\mathrm{rl}} \rho_{1}}{\mu_{1}}\right)_{\mathrm{nm}}\left(\frac{\mathrm{P}_{\mathrm{m}}-\mathrm{P}_{\mathrm{n}}}{\mathrm{D}_{\mathrm{nm}}}+\rho_{1} \mathrm{~g}_{\mathrm{nm}}\right)
$$

The subscript ' $\mathrm{nm}$ ' denotes appropriately weighted quantities pertaining to the interface between $\mathrm{n}$ and $\mathrm{m} . \mathrm{D}_{\mathrm{nm}}$ is the nodal distance, and $\mathrm{g}_{\mathrm{nm}}$ is the component of gravitational acceleration along the line from $m$ to $n . Q_{n}$ is the total sink/source rate in grid block $n$.

Let us now specialize to a 2-D vertical section with coordinates $\mathrm{x}$ (horizontal) and $\mathrm{z}$ (vertical), and consider the following simultaneous transformation of space and time coordinates,

$$
\begin{aligned}
& t \rightarrow t^{\prime}=\lambda_{t} \cdot t \\
& x \rightarrow x^{\prime}=\lambda_{x} \cdot x \\
& z \rightarrow z^{\prime}=\lambda_{z} \cdot z
\end{aligned}
$$

In this paper we strictly limit the discussion to the properties of Richards' equation under the scaling of Eq. (8). We do not address the broader issue of how the scaling of space coordinates may affect the appropriate averaging scales to be used for the definition of hydrologic properties of geologic media. Under the transformation Eq. (8), subdomain volumes $V_{n}$ scale by $\lambda_{x} \lambda_{z}$, so that the coefficient $\left(\Delta t / V_{n}\right)$ in front of flow and sink/source terms in Eq. (5) scales by $\lambda_{t} / \lambda_{x} \lambda_{z}$. Scaling behavior is different for horizontal and vertical areas, and is also different for gravity-driven flow as compared to capillary- or pressure-driven flow. Interface areas $A_{n m}$ for horizontal and vertical flow scale by $\lambda_{z}$ and $\lambda_{x}$, respectively. From Eq. (7) it is seen that the gravity (body force) flux term remains unchanged under the scaling Eq. (8), while pressure and capillary-driven fluxes scale with nodal distance $D_{n m}$ as $1 / \lambda_{x}$ and $1 / \lambda_{z}$ for horizontal and vertical components, respectively. The resulting scaling coefficients for horizontal and vertical components of flow terms corresponding to different flow mechanisms are shown in Table 3. 
We are interested in determining the conditions, if any, for which Eq. (5) would be invariant under the simultaneous space-and-time scaling of Eq. (8). For an invariance to hold, the scale factors in Eq. (8) must be chosen in such a way that the scaling coefficients given in Table 3

\section{Table 3. Scaling coefficients for flow equations.}

\begin{tabular}{|l|c|c|}
\hline \multicolumn{1}{|c|}{$\begin{array}{c}\text { flow component } \\
\text { flow mechanism }\end{array}$} & horizontal & vertical \\
\hline \hline capillarity, pressure & $\lambda_{\mathrm{t}} / \lambda_{\mathrm{x}}{ }^{2}$ & $\lambda_{\mathrm{t}} / \lambda_{\mathrm{z}}{ }^{2}$ \\
\hline gravity & -- & $\lambda_{\mathrm{t}} / \lambda_{\mathrm{z}}$ \\
\hline
\end{tabular}

are all equal to 1 . This would assure that changes $\Delta M_{n}$ in the accumulation terms calculated from Eq. (5) would be the same in the scaled as in the original system, grid block for grid block and time step for time step, so that fluid distributions would evolve in identical fashion. Inspection of Table 3 shows that it is not possible to achieve scaling invariance simultaneously for vertical flows under (capillary) pressure and gravity forces. Indeed, the former would demand $\lambda_{t} / \lambda_{z}{ }^{2}=1$, whereas the latter would require $\lambda_{1} / \lambda_{z}=1$, which conditions can be satisfied simultaneously only for the trivial case $\lambda_{z}=1$. Therefore, generally speaking, flow processes involving these different driving forces will not possess any scaling invariance but will evolve differently on different scales. However, an approximate scaling invariance may still hold when liquids percolate downward in an unsaturated medium of "relatively high" permeability. In this case (sub-)vertical flows will be primarily driven by gravity, and capillary and pressure effects on vertical flows may be small. An approximate invariance would then be expected to hold when scaling in such a fashion that $\lambda_{t} / \lambda_{x}{ }^{2}=1$ and $\lambda_{t} / \lambda_{z}=1$, i.e.,

$$
\lambda_{\mathrm{t}}=\lambda_{\mathrm{x}}^{2}=\lambda_{\mathrm{z}}
$$

Thus, the vertical length scale and the time scale need to be stretched by the square of the horizontal scale factor. Under the scaling of Eq. (9), vertical flow contributions from capillary and pressure effects would not be invariant, but would change by a factor $\lambda_{t} / \lambda_{z}{ }^{2}=1 / \lambda_{z}=1 / \lambda_{x}{ }^{2}$. This relationship shows that, if indeed capillary and pressure effects on vertical flows are small in the initial (unscaled) system, they will be even smaller under upscaling $\left(\lambda_{x}>1\right)$. However, under downscaling $\left(\lambda_{x}<1\right)$ these terms will increase in magnitude relative to the gravity term, suggesting that the approximate scaling invariance of Eq. (9) will eventually break down when scaling down to "sufficiently" small systems. 
A number of numerical simulation experiments have been performed to test the approximate scaling invariance postulated in Eq. (9). We applied scale factors of $\lambda_{x}=5$ and $1 / 5$, respectively, simultaneously scaling spatial dimensions and time according to Eq. (9). The spatial scaling changes the $20 \mathrm{~m} \times 20 \mathrm{~m}$ heterogeneous fracture of Fig. 2 into $100 \mathrm{~m} \times 500 \mathrm{~m}\left(\lambda_{\mathrm{x}}=5\right)$ and $4 \mathrm{~m} \times$ $0.8 \mathrm{~m}\left(\lambda_{\mathrm{x}}=1 / 5\right)$, respectively. Source (injection) rates were scaled by $\lambda_{\mathrm{t}} / \lambda_{\mathrm{x}} \lambda_{z}=1 / \lambda_{\mathrm{x}}$ (cf. the discussion following Eq. (8)). Results are shown in Figs. 15 and 16, and Table 4. Upscaling by a factor $\lambda_{x}=5$ produces seepage patterns that are so close to the original as to be virtually indistinguishable in all cases (compare Figs. 6 and 15). The almost exact mimicking of even smallscale details in saturation distributions between the original and the scaled system demonstrates the

Table 4. Scaling behavior of simulated seeps.

\begin{tabular}{|c|c|c|c|c|}
\hline Seep & $\# 1$ & $\# 2$ & $\# 3$ & $\# 4$ \\
\hline \multicolumn{5}{|l|}{ Upscaling by $\lambda_{x}=5.0$} \\
\hline \begin{tabular}{|l|} 
Breakthrough time at \\
$487.5 \mathrm{~m}$ depth (days) \\
\end{tabular} & 15.87 & 14.84 & 24.95 & 16.53 \\
\hline Average seepage velocity $(\mathrm{m} / \mathrm{hr})$ & 1.28 & 1.37 & 0.81 & 1.23 \\
\hline \multicolumn{5}{|l|}{ Downscaling by $\lambda_{x}=0.2$} \\
\hline $\begin{array}{l}\text { Breakthrough time at } \\
0.78 \mathrm{~m} \text { depth ( } \mathrm{min})\end{array}$ & 55.67 & 51.95 & 71.65 & 58.14 \\
\hline Average seepage velocity $(\mathrm{m} / \mathrm{hr})$ & 0.84 & 0.90 & 0.65 & 0.80 \\
\hline
\end{tabular}

excellent validity of the approximate scaling relationship Eq. (9) for this case. This is also borne out by a comparison of the average seepage velocities, see Tables 2 and 4 . A different picture emerges for downscaling by a factor of $\lambda_{x}=1 / 5$. Comparison of Fig. 16 with Fig. 6 shows that, while overall seepage patterns are similar, there are very visible and significant differences. Regions of large water saturation tend to be more extensive in the downscaled system, and overall water saturations tend to be larger. This leads to seepage velocities that are typically $30 \%$ slower (see Table 4). It is evident that capillary effects on vertical flow have become significant for the small-scale system shown in Fig. 16, so that the scaling relationship Eq. (9) is a rather poor approximation in this case. 


\section{Validity of Scaling Invariance}

We now attempt to quantify the limitations of the approximate scaling invariance, Eq. (9). Consider gravity-driven seepage in a homogeneous fracture inclined at an angle $\alpha$ relative to the vertical. From Darcy's law, in a fracture with vertical permeability $k_{v}$ the downward advancement of a seep under unit head gradient conditions will proceed with a velocity

$$
\mathrm{v}=\frac{\mathrm{k}_{\mathrm{v}}}{\phi} \frac{\rho_{1} g \cos \alpha}{\mu_{1}} \frac{\mathrm{k}_{\mathrm{rl}}}{\left(\mathrm{S}_{1}-\mathrm{S}_{\mathrm{lr}}\right)}
$$

In writing Eq. (10) we have assumed that the fracture initially is at irreducible water saturation $S_{\mathrm{lr}}$. As the seep advances it is affected by capillary forces. These are diffusive in nature, corresponding to second-order space derivatives in the governing Eq. (2). Over a time period $t$, diffusive propagation occurs over a distance (Carslaw and Jaeger, 1959)

$$
l=\sqrt{\mathrm{Dt}}
$$

where

$$
D=\frac{k_{r e f} k_{r l}}{\phi \mu_{1}} \frac{d P_{c a p}}{d S_{1}}
$$

is the effective unsaturated diffusivity at reference permeability $k_{\text {ref }}$ (Pruess et al., 1990b). The time required for a seep to advance over a domain of vertical length $L$ is $t=L / v$. The diffusive propagation from capillary effects during this time will be given by

$$
l=\sqrt{\mathrm{DL} / \mathrm{v}}
$$

In order for scaling invariance to hold, we require that $l / \mathrm{L}<1$, or

$$
\frac{l}{L}=\sqrt{\frac{\mathrm{D}}{\mathrm{vL}}} \ll 1
$$

The group $\mathrm{D} / \mathrm{vL}$ is an inverse Peclet number. Both diffusivity $\mathrm{D}$ and advective velocity $\mathrm{v}$ increase strongly with increasing liquid saturation (Figs. 17, 18). For the homogeneous fracture the seepage velocity was approximately $1 \mathrm{~m} / \mathrm{hr}$ (Fig. 5), which according to Fig. 18 is reached at a liquid saturation of $S_{1}=0.62$. This is considerably larger than the average saturation of the 
homogeneous seep (cf. Fig. 9), but it is consistent with the results shown in Fig. 5; indeed, downward advancement of the seep is determined by the inner region of larger $S_{1}$. From Eqs. (10, 12), the group $D / v$ for a vertical fracture is given by

$$
\frac{D}{v}=\frac{k_{r e f}}{k_{v}} \frac{S_{1}-S_{1 r}}{\rho_{1} g} \frac{d P_{c a p}}{d S_{l}}
$$

which for the parameters chosen here (see Table 1) is only weakly dependent on saturation in the range $0.2 \leq S_{1} \leq 0.95$ (Fig. 19). Using a typical (and insensitive) value of $D / v=0.11 \mathrm{~m}$ at the saturation level of interest, the parameter group of Eq. (14) takes on the values shown in Table 5 for the three scaled fractures.

Table 5. Scale dependence of capillary to gravity effects on seepage.

\begin{tabular}{|c|c|c|}
\hline$\lambda_{x}$ & $L(m)$ & $l / L=\sqrt{D / v L}$ \\
\hline \hline 0.2 & 0.8 & 0.371 \\
\hline 1 & 20 & 0.074 \\
\hline 5 & 500 & 0.015 \\
\hline
\end{tabular}

It is seen that the ratio of capillary to gravity effects is small $(l / \mathrm{L} \ll 1)$ for $\lambda_{x}=1$ and 5 , but not for $\lambda_{\mathrm{x}}=0.2$, which explains the observed scale invariance for the former, and the lack of scale invariance for the latter.

The above derivation and discussion emphasized the time-dependent competition between gravity and capillary effects on downward advective flows, and is only applicable to transient behavior. Continued application of infiltration at the top of the fracture, beyond the time required for the seep to break through at the bottom boundary, will cause the seepage pattern to change (see Fig. 12) in ways that will not be invariant under the constraint Eq. (9). Indeed, as steady state is approached time will no longer be a factor, rendering Eq. (9) inapplicable.

In closing we emphasize that the scaling relationship Eq. (9), where applicable, will hold only for media with self-affine heterogeneity structure, where the heterogeneous permeability fields scale by factors $\lambda_{x}$ and $\lambda_{z}$ in horizontal and vertical directions, respectively. Practically, a mathematically rigorous scaling of heterogeneous permeability fields with $\lambda_{z}=\lambda_{x}{ }^{2}$ could possibly be achieved for a man-made machined specimen, but will never be realized for natural fractures. 
However, many naturally occurring geometries are self-similar or self-affine fractals (Mandelbrot, 1983), and rock fractures may also have such properties (Brown and Scholz, 1985; Wang et al., 1988; Nolte et al., 1989). Heterogeneous fractures on different scales may represent different realizations of the same or a similar heterogeneity structure. Accordingly, for natural fracture systems Eq. (9) should be interpreted in a conceptual rather than a quantitative sense, suggesting a possible relationship between transient seepage patterns on different scales.

\section{Discussion and Conclusions}

Recent advances in numerical simulation capabilities make possible fully transient simulations of unsaturated flow in highly heterogeneous media, with detailed resolution of smallscale heterogeneity, including transitions between partially and fully saturated conditions locally. We have simulated a small number of seeps (4) in a stochastically homogeneous medium, observing a range of behavior. The simulations presented here were exploratory in nature; a much larger number of realizations should be studied in the future to more fully explore the behavior of seeps and determine their statistical features.

Our limited number of simulations of localized infiltration in a synthetic heterogeneous fracture has demonstrated many of the features seen in naturally fractured systems in thick unsaturated zones, such as at Rainier Mesa (Lawrence Berkeley Laboratory, 1991), including flow fingering, bypassing, and ponding. The examples studied here suggest that localized infiltration can give rise to a variety of behavior, from localized seeps to broad plumes with dispersed flow at some depth beneath the infiltration region. Field experiments have shown that flow focussing or funneling is possible for seepage in partially saturated heterogeneous media (Kung, 1990a, b). The exploration of heterogeneity conditions for which spatially-distributed infiltration could give rise to highly localized preferential flows is of considerable practical interest, as such flows could provide fast paths for the migration of solutes.

An approximate invariance of seepage under simultaneous space-and-time scaling was theoretically derived. The validity and limitations of this invariance were demonstrated through numerical simulations, and through analyses of capillary- and gravity-driven flows.

Future experimental and numerical studies should investigate different types of heterogeneity structures and a variety of boundary and source conditions, including non-uniform, localized infiltration at time-varying rates. Of interest is also the interaction between seeps, and between fractures and matrix rock. 


\section{QA Notice for Yucca Mountain Project}

Software used in the analyses reported here includes (i) TOUGH2, Version 1.11 (qualified), (ii) a special fluid property module "EOS9" for TOUGH2 (not qualified), (iii) enhanced versions of several TOUGH2 program units for strongly heterogeneous systems (not qualified), and (iv) a program for the turning bands method, written by Andrew Tompson of LLNL (not qualified). No qualified data were used in the calculations. Therefore, the results presented in this report cannot be considered as qualified.

\section{Acknowledgement}

This work was supported, in part, by the Director, Office of Civilian Radioactive Waste Management, and by the Director, Office of Energy Research, Office of Health and Environmental Sciences, Biological and Environmental Research Program, of the U.S. Department of Energy under Contract No. DE-AC03-76SF00098. Thanks are due to Emilio Antunez for his help with the generation of spatially correlated stochastic fields, and to Jil Geller and Boris Faybishenko for a review of the manuscript and the suggestion of improvements.

\section{References}

Abelin, H., L. Birgersson, J. Gidlund, L. Moreno, I. Neretnieks and S. Tunbrant. Results from Some Tracer Experiments in Crystalline Rocks in Sweden, Coupled Processes Associated with Nuclear Waste Respositories, C.F. Tsang, (ed.), Academic Press, Inc., New York, 1987.

Brown, S. R. and C. H. Scholz. Broad Bandwidth Study of the Topography of Natural Rock Surfaces, J. Geophy. Res., 90, 12,575-12,582, 1985.

Carslaw, H. S., and J. C. Jaeger. Conduction of Heat in Solids, Oxford University Press, Oxford, England, Second Edition, 1959.

Chesnut, D.A. Characterizing the Altered Zone at Yucca Mountain: The Beginning of a Testing Strategy, Proceedings, Third High Level Radioactive Waste Management International Conference, Las Vegas, NV, Vol. 1, pp. 1026 - 1039, American Nuclear Society, La Grange Park, IL, April 12-16, 1992.

Chesnut, D.A. Dispersivity in Heterogeneous Permeable Media, Proceedings, Fifth Annual International High-Level Radioactive Waste Management Conference, Las Vegas, NV, Vol. 4, pp. 1822 - 1841, American Nuclear Society, La Grange Park, IL, May 1994.

Eaton, R.R., C.K. Ho, R.J. Glass, M.J. Nicholl, and B.W. Arnold. Modeling of Flow through Fractured Tuff at Fran Ridge, Proceedings, Seventh Annual International High-Level Radioactive Waste Management Conference, Las Vegas, NV, pp. 76 - 78, American Nuclear Society, La Grange Park, IL, April 1996.

Firoozabadi, A. and J. Hauge. Capillary Pressure in Fractured Porous Media, Journal of Petroleum Technology, 784-791, June 1990. 
Gauthier, J.H., M.L. Wilson, and F.C. Lauffer. Estimating the Consequences of Significant Fracture Flow at Yucca Mountain, Proceedings, Third Annual International High-Level Radioactive Waste Management Conference, Las Vegas, NV, Vol. 1, pp. 891 - 898, American Nuclear Society, La Grange Park, IL, April 1992.

Gauthier, J.H. An Updated Fracture Flow Model for Total-System Performance Assessment of Yucca Mountain, Proceedings, Fifth Annual International High-Level Radioactive Waste Management Conference, Las Vegas, NV, Vol. 3, pp. 1663 - 1670, American Nuclear Society, La Grange Park, IL, May 1994.

Geller, J. and K. Pruess. On Water Infiltration in Rough-Walled Fractures. Proceedings, Sixth Annual International High-Level Radioactive Waste Management Conference, Las Vegas, NV, pp. 23 - 25, American Nuclear Society, La Grange Park, Il, May 1995.

Glass, R.J. Modeling Gravity-Driven Fingering in Rough-Walled Fractures Using Modified Percolation Theory, Fourth Annual International High-Level Radioactive Waste Management Conference, Las Vegas, NV, pp. 2042 - 2052, American Nuclear Society, La Grange Park, II, April 1993.

Kung, K. J. S. Preferential Flow in a Sandy Vadose Zone: 1. Field Observation, Geoderma, Vol. 46 , pp. 51-58, 1990 a.

Kung, K. J. S. Preferential Flow in a Sandy Vadose Zone: 2. Mechanism and Implications, Geoderma, Vol. 46, pp. 59-71, $1990 \mathrm{~b}$.

Lawrence Berkeley Laboratory, Geologic Repository Project, A Review of Rainier Mesa Tunnel and Borehole Data and their Possible Implications to Yucca Mountain Site Study Plans, Lawrence Berkeley Laboratory Report LBL-32068, 99p., December 1991.

Leverett, M. C., Capillary Behavior in Porous Solids, Trans. Soc. Pet. Eng. AIME, 142, 152$169,1941$.

Liu, B., J. Fabryka-Martin, A. Wolfsberg, B. Robinson and P. Sharma. Significance in Apparent Discrepancies in Water Ages Derived From Atmospheric Radionuclides at Yucca Mountain, Nevada, Los Alamos National Laboratory Report LA-UR-95-572, Los Alamos, NM, February 1995.

Mandelbrot, B.B. The Fractal Geometry of Nature, W.H. Freeman and Co., New York, NY 1983.

Moridis, G. and K. Pruess. Flow and Transport Simulations Using T2CG1, a Package of Conjugate Gradient Solvers for the TOUGH2 Family of Codes, Lawrence Berkeley Laboratory Report LBL-36235, Lawrence Berkeley Laboratory, Berkeley, CA, 1995.

Nativ, R., E. Adar, O. Dahan and M. Geyh. Water Recharge and Solute Transport through the Vadose Zone of Fractured Chalk under Desert Conditions, Water Resour. Res., Vol. 31, No. 2, pp. 253 - 261, February 1995.

Nicholl, M.J., R.J. Glass, and H.A. Nguyen. Wetting Front Instability in an Initially Wet Unsaturated Fracture. Proceedings, Fourth High Level Radioactive Waste Management International Conference, Las Vegas, Nevada, April 26-30, 1993.

Nicholl, M.J., R.J. Glass, and S.W. Wheatcraft. Gravity-Driven Infiltration Instability in Initially Dry Nonhorizontal Fractures. Water Res. Res., Vol. 30, No. 9, pp. 2533-2546, 1994. 
Nitao, J.J. and T.A. Buscheck. Infiltration of a Liquid Front in an Unsaturated, Fractured Porous Medium, Water Resour. Res., Vol. 27, No. 8, pp. 2099 - 2112, 1991.

Nolte, D.D., L.J. Pyrak-Nolte and N.G.W. Cook. The Fractal Geometry of the Flow Paths in Natural Fractures in Rock and the Approach to Percolation, Pageoph, Vol. 131, Nos. 1/2, pp. 111-138, 1989.

Oldenburg, C. M. and K. Pruess. On Numerical Modeling of Capillary Barriers, Water Resour. Res., Vol. 29, No. 4, pp. 1045-1056, 1993.

Persoff, P. and K. Pruess. Two-Phase Flow Visualization and Relative Permeability Measurement in Natural Rough-Walled Rock Fractures, Water Resour. Res., Vol. 31, No. 5, pp. 11751186, May 1995.

Peters, R. R. and E. A. Klavetter. A Continuum Model for Water Movement in an Unsaturated Fractured Rock Mass, Water Resour. Research, 24 (3), 416-430, 1988.

Pruess, K. TOUGH2 - A General Purpose Numerical Simulator for Multiphase Fluid and Heat Flow, Report No. LBL-29400, Lawrence Berkeley Laboratory, Berkeley, CA, May 1991.

Pruess, K. On the Validity of a Fickian Diffusion'Model for the Spreading of Liquid Infiltration Plumes in Partially Saturated Heterogeneous Media. Computational Methods in Water Resources X, Vol. 1, pp. 537 - 544, Kluwer Academic Publishers, Dordrecht, Boston, London, 1994.

Pruess, K. Effective Parameters, Effective Processes: From Porous Flow Physics to In Situ Remediation Technology. Invited paper, presented at VEGAS-Symposium, University of Stuttgart/Germany, September 25-27, 1995 (also: Lawrence Berkeley Laboratory Report LBL-37414)

Pruess, K. A Fickian Diffusion Model for the Spreading of Liquid Plumes Infiltrating in Heterogeneous Media. in press in Transport in Porous Media, 1996.

Pruess, K. and E. Antunez. Applications of TOUGH2 to Infiltration of Liquids in Media with Strong Heterogeneity, Proceedings of the TOUGH Workshop '95, Lawrence Berkeley Laboratory Report LBL-37200, pp. 69-76, Berkeley, CA, March 1995.

Pruess, K., and Y. W. Tsang. On Two-Phase Relative Permeability and Capillary Pressure of Rough-Walled Rock Fractures, Water Resour. Res., Vol. 26, No. 9, pp. 1915-1926, September 1990.

Pruess, K., J. S. Y. Wang and Y. W. Tsang. On Thermohydrological Conditions Near HighLevel Nuclear Wastes Emplaced in Partially Saturated Fractured Tuff. Part 1. Simulation Studies with Explicit Consideration of Fracture Effects, Water Res. Res., 26 (6), 12351248, 1990a.

Pruess, K., J. S. Y. Wang and Y. W. Tsang. On Thermohydrological Conditions Near HighLevel Nuclear Wastes Emplaced in Partially Saturated Fractured Tuff. Part 2. Effective Continuum Approximation, Water Res. Res., 26 (6), 1249-1261, $1990 \mathrm{~b}$.

Tompson, A.F.B. Implementation of the Three-Dimensional Turning Bands Random Field Generator, Water Resour. Res., Vol. 25, No. 10, pp. 2227 - 2243, 1989. 
van Genuchten, M. Th. A Closed-Form Equation for Predicting the Hydraulic Conductivity of Unsaturated Soils, Soil Sci. Soc. Am. J., Vol. 44, pp. 892-898, 1980.

Witherspoon, P. A., J. S. Y. Wang, K. Iwai, and J. E. Gale. Validity of Cubic Law for Fluid Flow in a Deformable Rock Fracture, Water Resour. Res., 16 (6), 1016-1024, 1980.

Wang, J.S.Y. and T.N. Narasimhan. Hydrologic Mechanisms Governing Fluid Flow in a Partially Saturated, Fractured, Porous Medium, Water Resour. Res., 21 (12), 1861-1874, December 1985.

Wang, J.S.Y., T.N. Narasimhan, and C.H. Scholz. Aperture Correlation of a Fractal Fracture, Journal of Geophysical Res., 93 (B3), 2216-2224, 1988.

Wang, J.S.Y., N.G.W. Cook, H.A. Wollenberg, C.L. Carnahan, I. Javandel, and C.F. Tsang. Geohydrologic Data and Models of Rainier Mesa and Their Implications to Yucca Mountain, Proceedings, Fourth Annual International High-Level Radioactive Waste Management Conference, Las Vegas, NV, Vol. 1, pp. 675 - 681, American Nuclear Society, La Grange Park, II, April 1993.

Wang, J.S.Y: and T.N. Narasimhan. Unsaturated Flow in Fractured Porous Media, in: Flow and Contaminant Transport in Fractured Rock, Academic Press, 1993. 


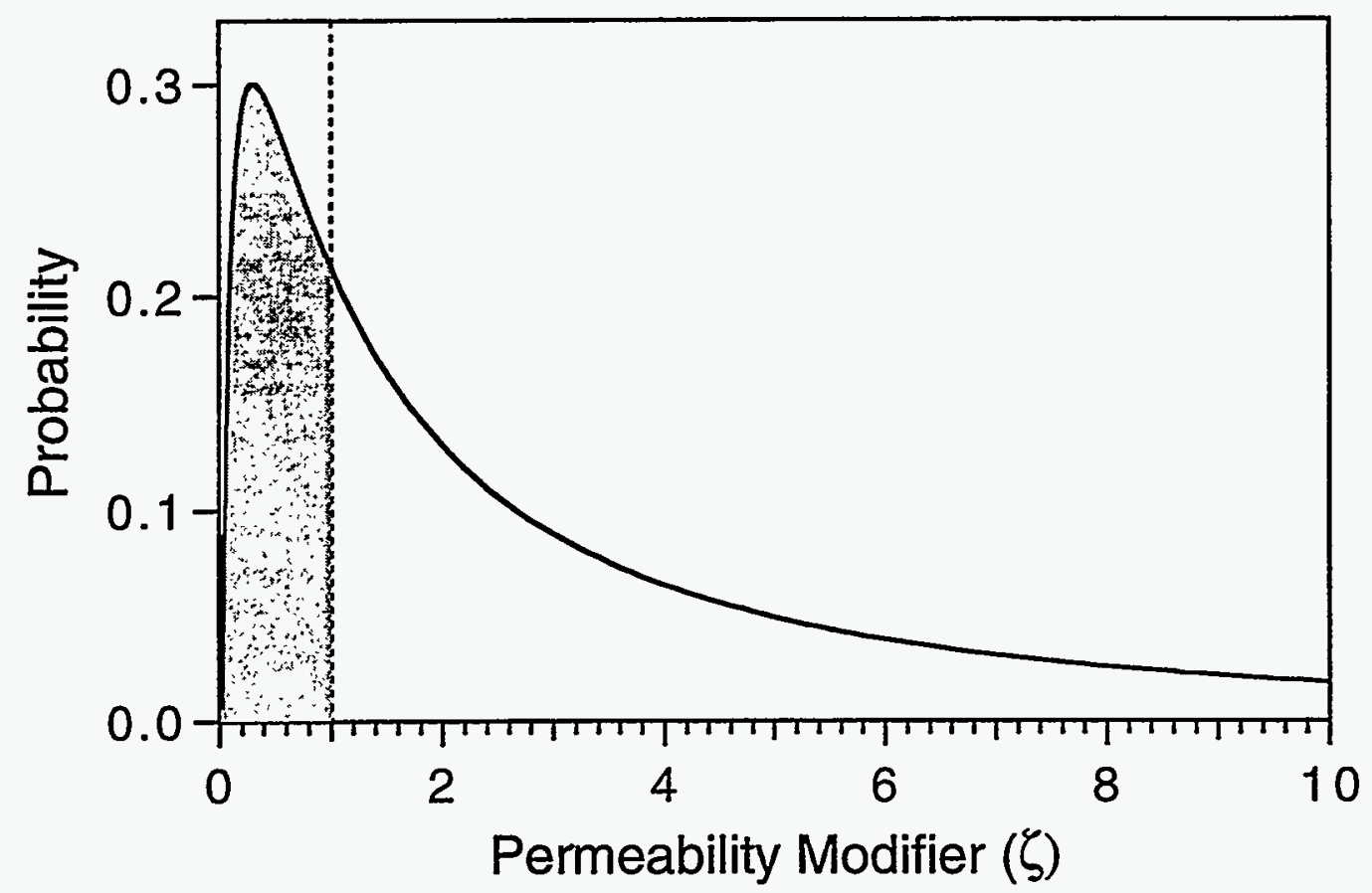

Figure 1. Lognormal distribution of permeability modification coefficients for synthetic fracture. The shaded region, having an area of 0.264 , ends up representing asperity contacts after application of the shift given by Eq. (4). 

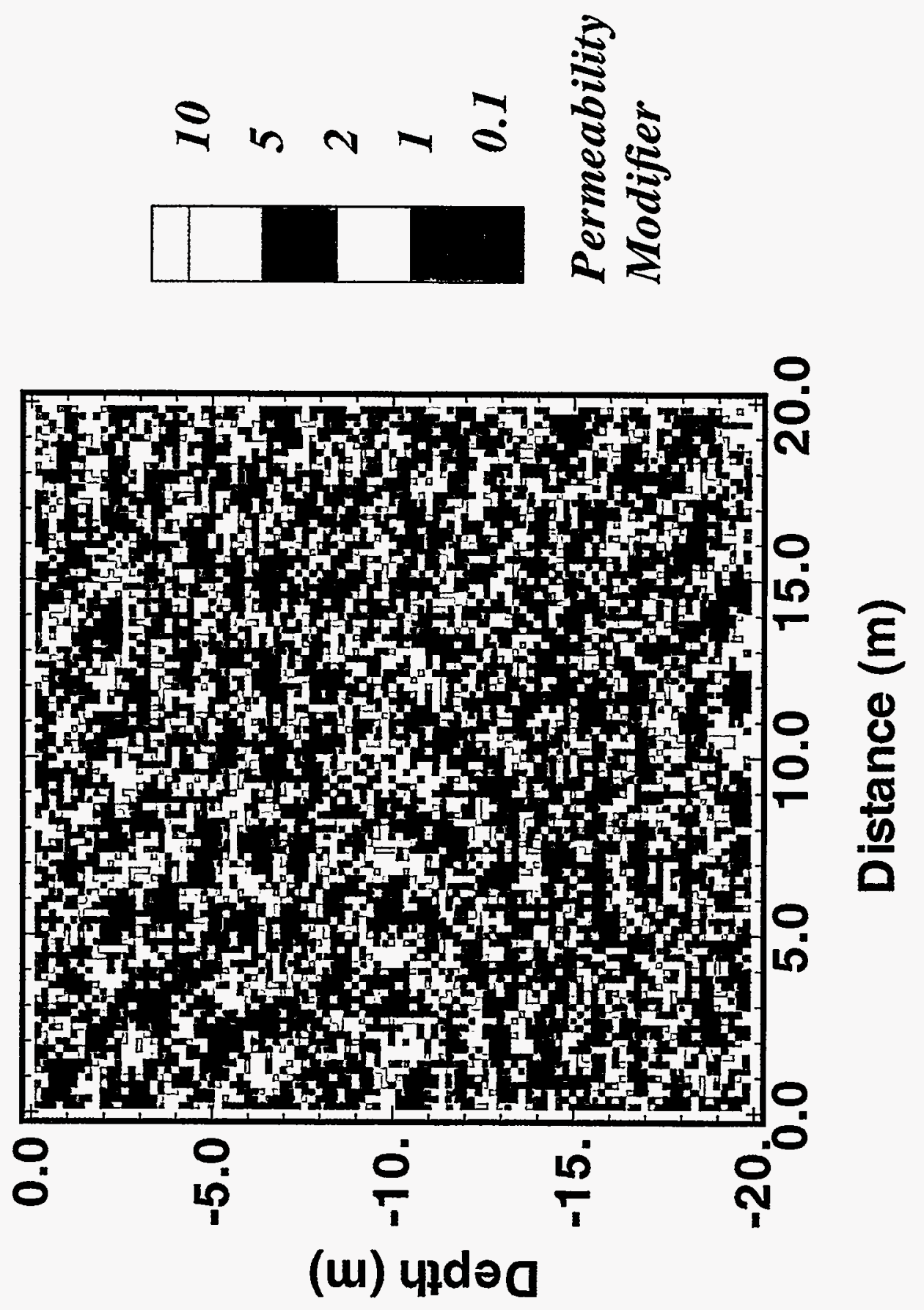

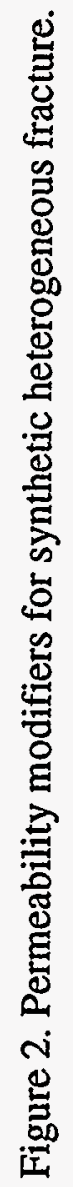




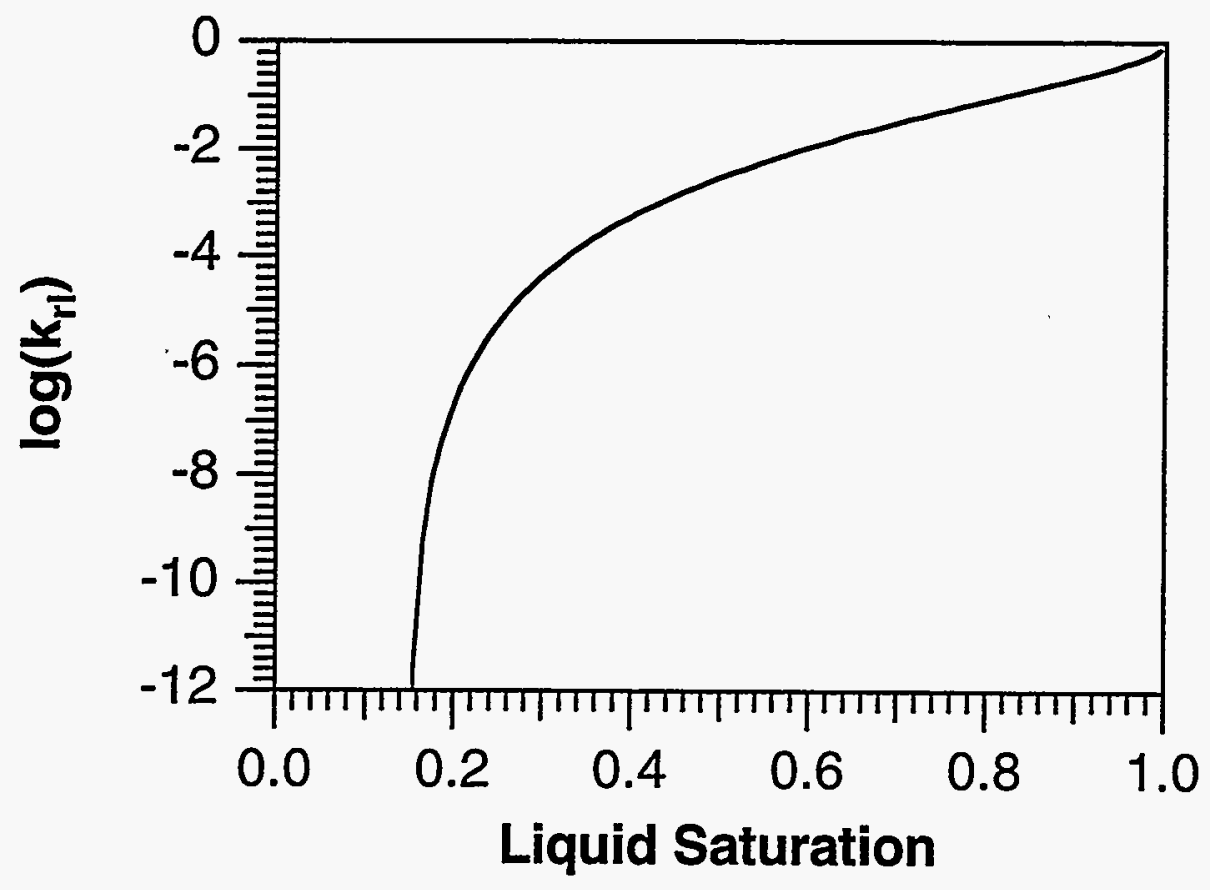

Figure 3. Relative permeability function.

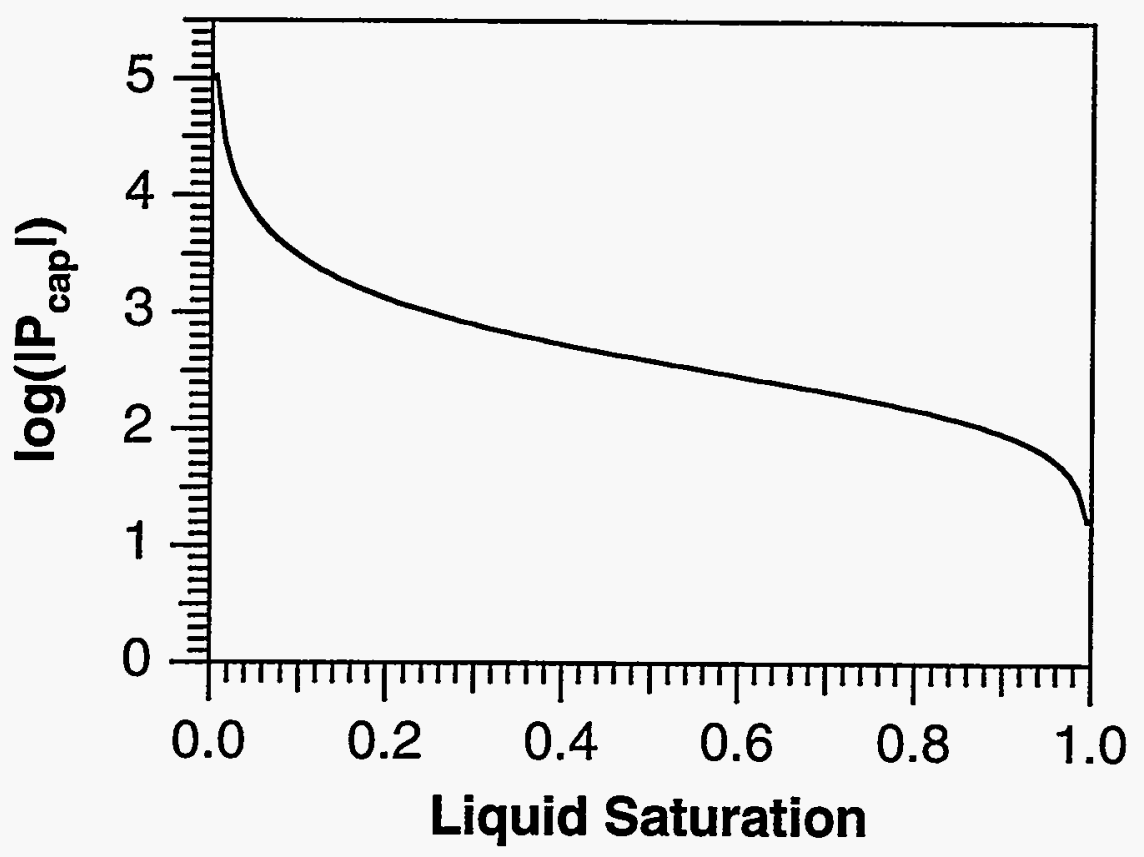

Figure 4. Capillary pressure function for reference permeability of $\mathrm{k}=10^{-9} \mathrm{~m}^{2}$. Capillary pressure is in units of Pascals. 

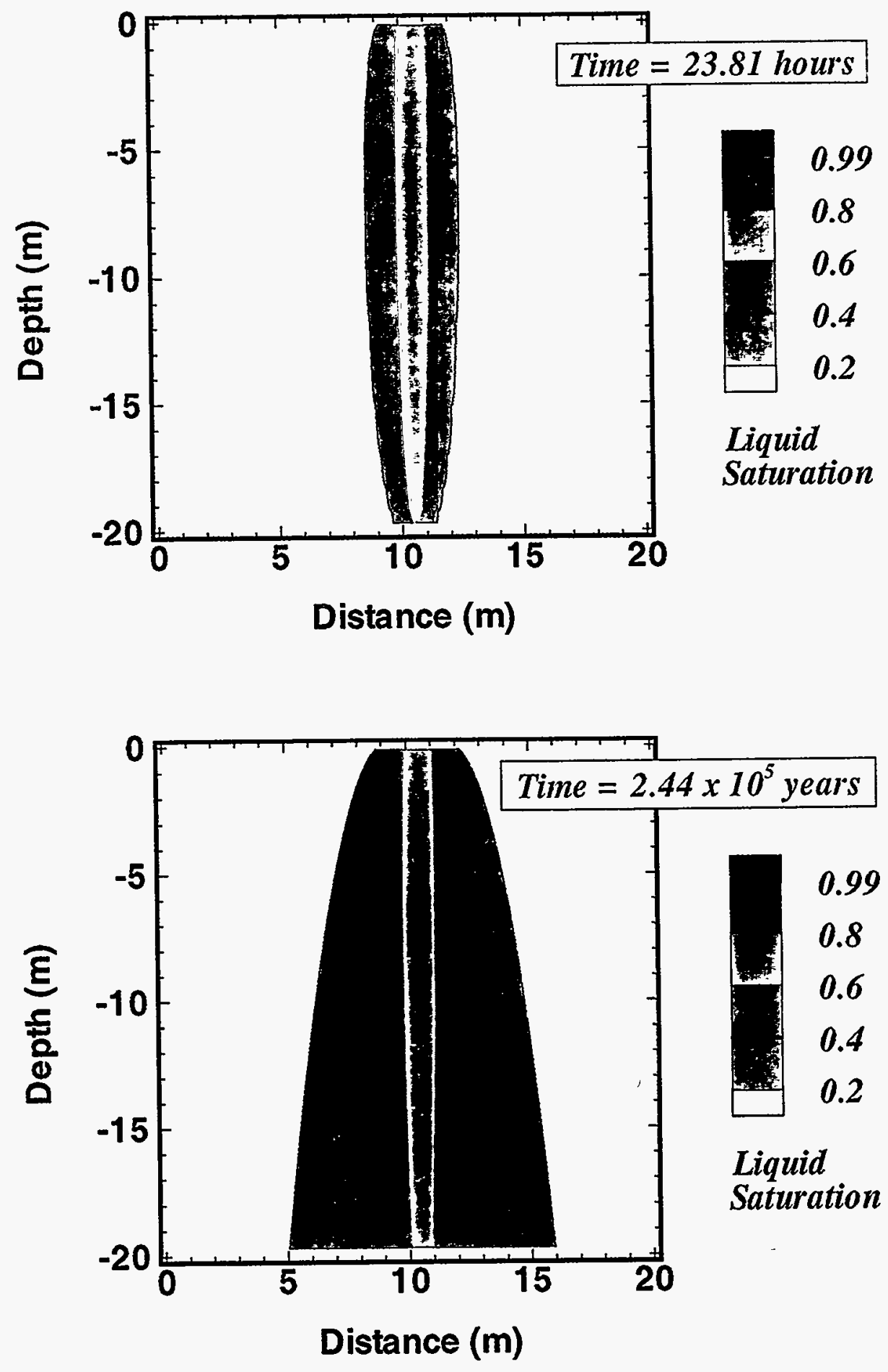

Figure 5. Liquid seeps for injection into a homogeneous fracture at a constant rate of $10^{-3} \mathrm{~kg} / \mathrm{s}$ over a $1 \mathrm{~m}$ interval at the top, centered at $10.5 \mathrm{~m}$. Horizontal and vertical permeability are, respectively, $0.6911 \times 10^{-9} \mathrm{~m}^{2}$ and $0.36087 \times 10^{-9} \mathrm{~m}^{2}$, corresponding to the average permeabilities of the heterogeneous fracture of Fig. 2. Seeps are shown at the time of breakthrough (top) and at $2.44 \times 10^{5}$ years (bottom) when saturation conditions are near steady state. 

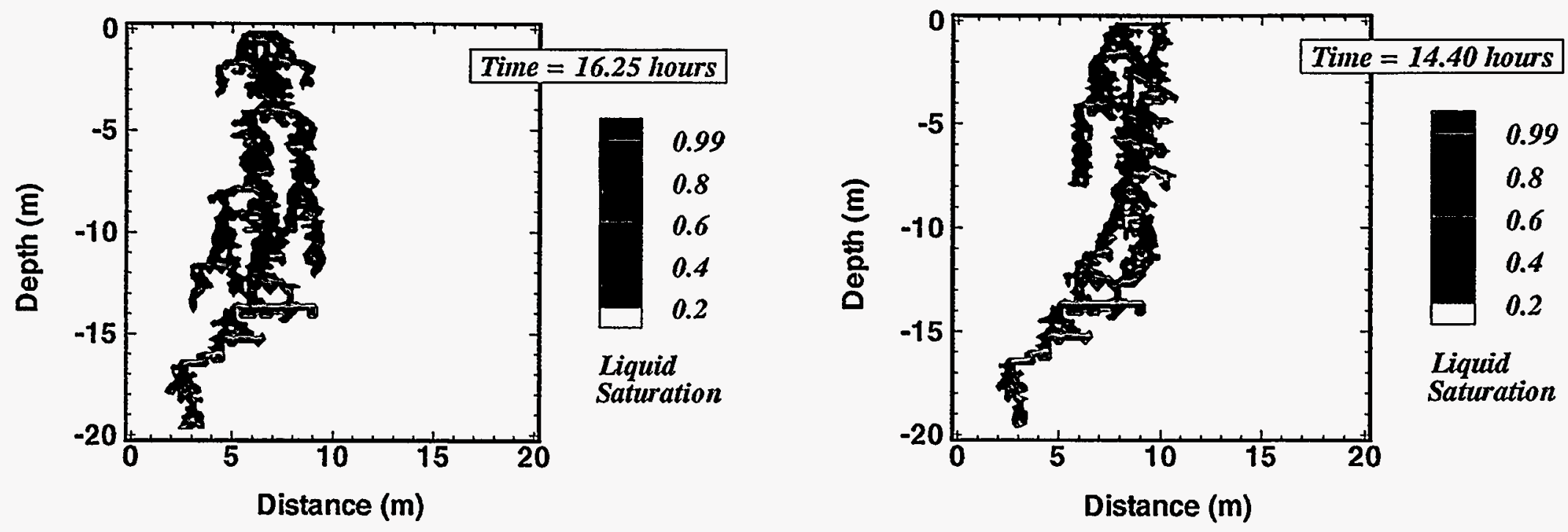

N
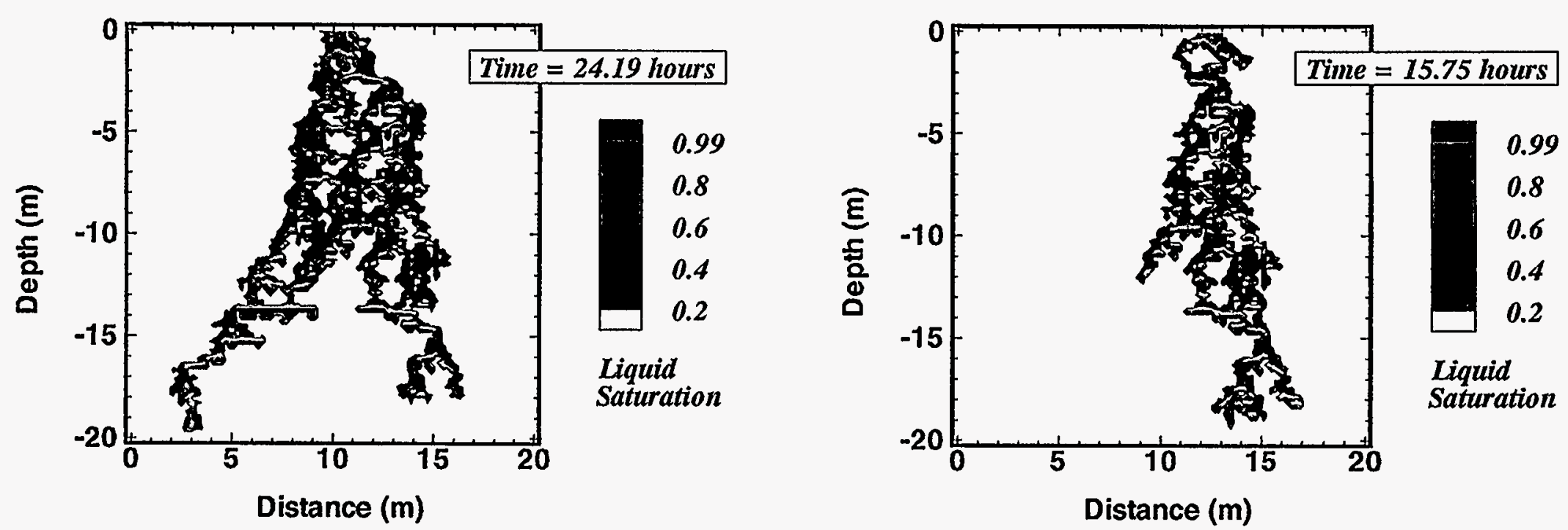

Figure 6. Liquid seeps shown at the time of breakthrough at a depth of $19.5 \mathrm{~m}$. Water is injected at a constant rate of $10^{-3} \mathrm{~kg} / \mathrm{s}$ over a $1 \mathrm{~m}$ interval at the top of the fracture. The injection interval is centered at different distances from the left boundary, namely, $6.5 \mathrm{~m}$ (top left), $8.5 \mathrm{~m}$ (top right), $10.5 \mathrm{~m}$ (bottom left), and $12.5 \mathrm{~m}$ (bottom right). Reference permeability is $10^{-9} \mathrm{~m}^{2}$. 


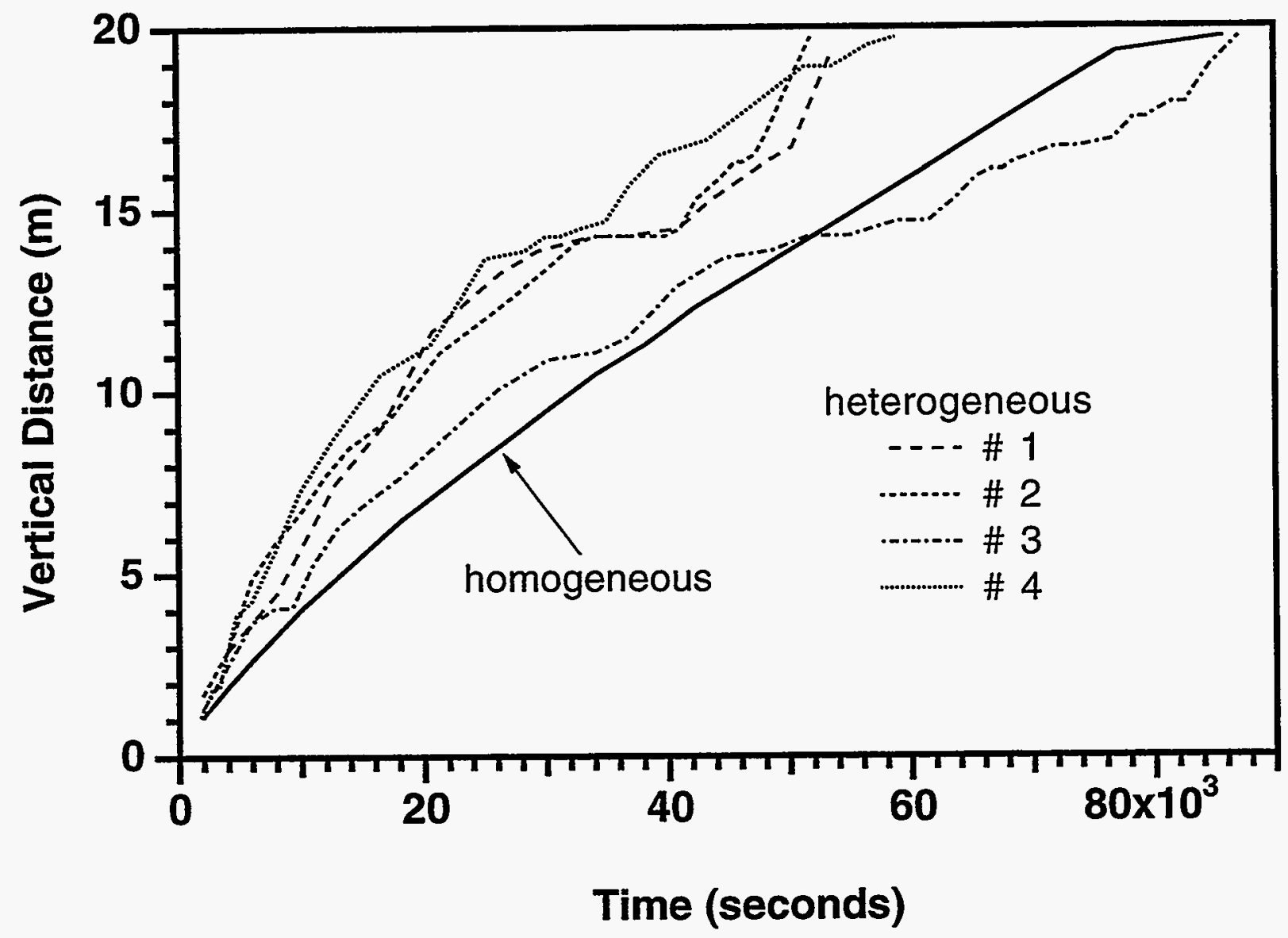

Figure 7. Vertical advancement of seeps with time for injection into a homogeneous and a heterogeneous fracture. The heterogeneous cases labeled \# 1 through 4 correspond to the sequence of four seeps shown in Fig. 6, with injection intervals centered at $6.5,8.5,10.5$, and $12.5 \mathrm{~m}$, respectively. 


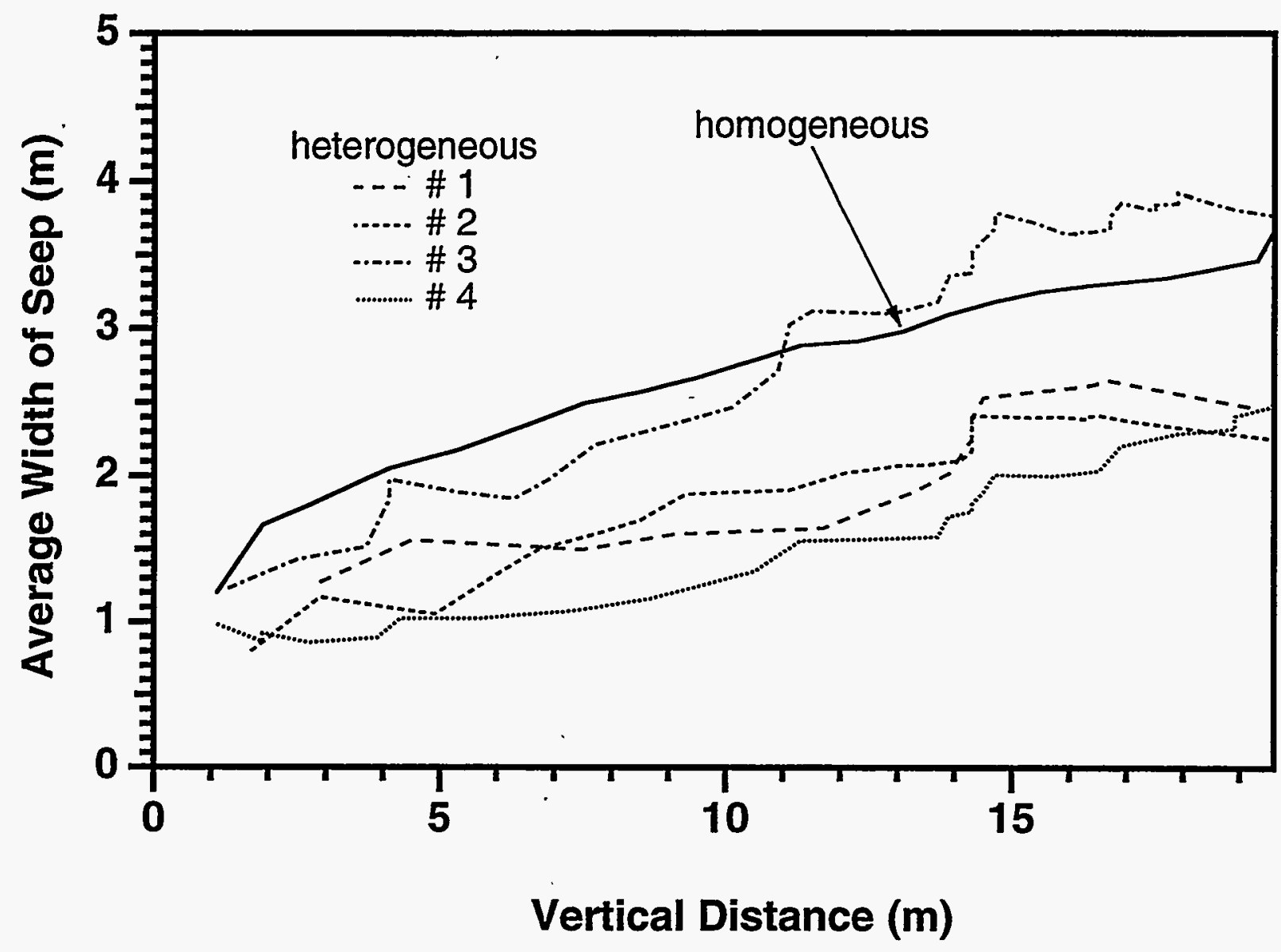

Figure 8. Average width of the five seeps of Fig. 7 as function of vertical travel distance. Average width is defined as the ratio of fracture wall area contacted by the seep to vertical travel distance. 


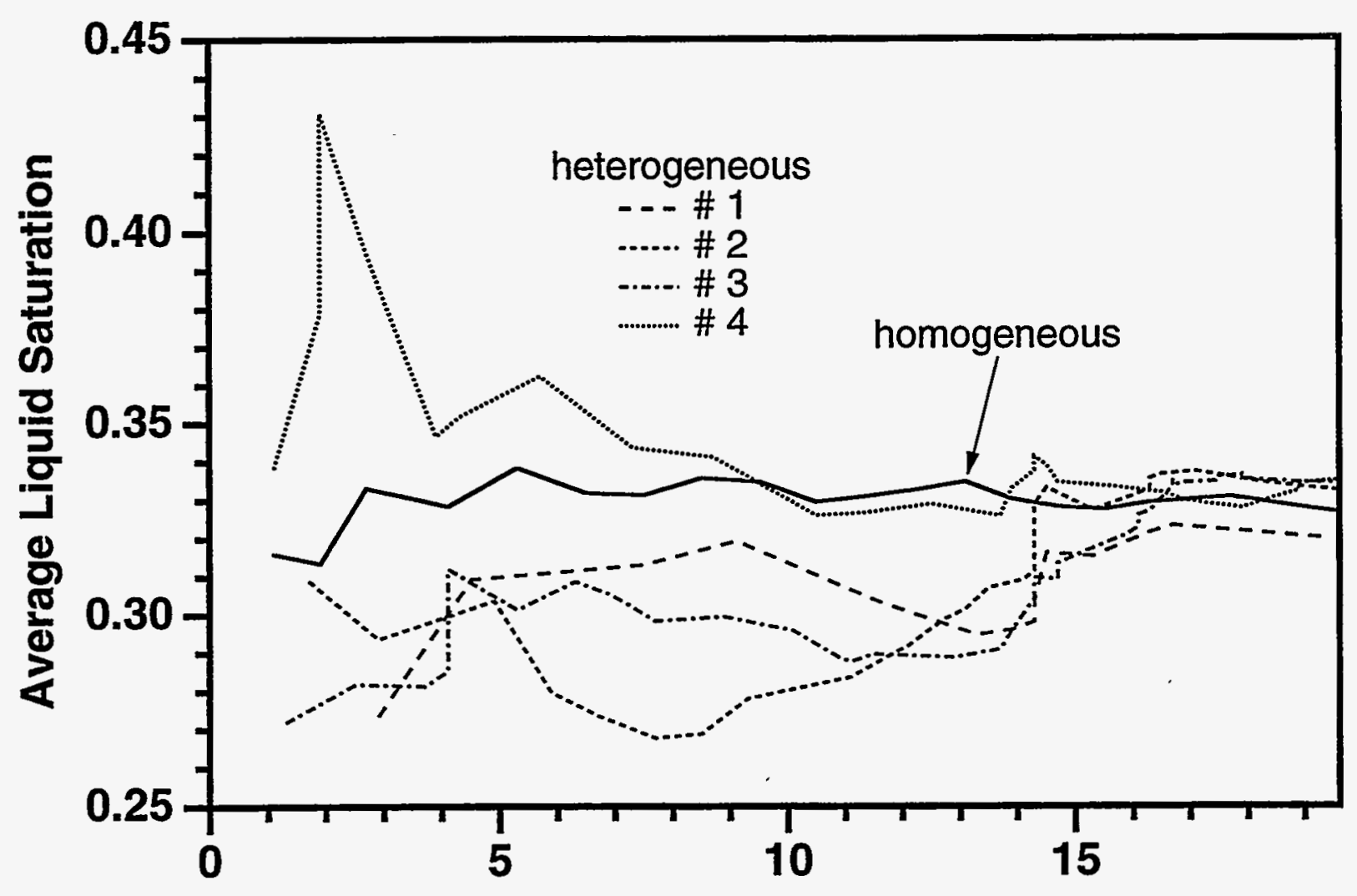

Vertical Distance (m)

Figure 9. Average liquid saturation of the five seeps of Fig. 7 as function of vertical travel distance. Liquid saturation is the ratio of liquid volume in the seep to the total pore volume in the region swept by the seep. 

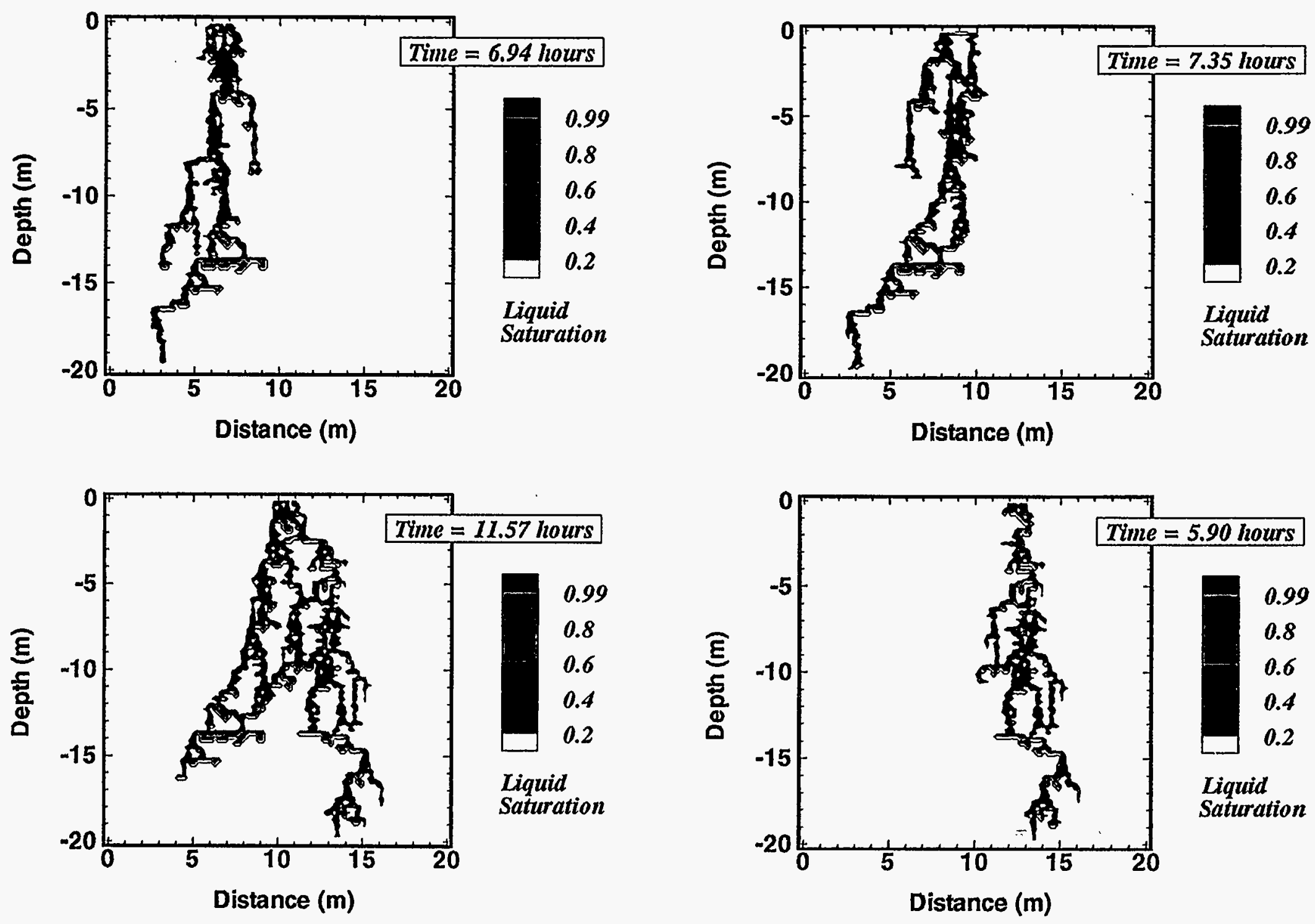

Figure 10. As Fig. 6, but reference permeability of $10^{-7} \mathrm{~m}^{2}$. 

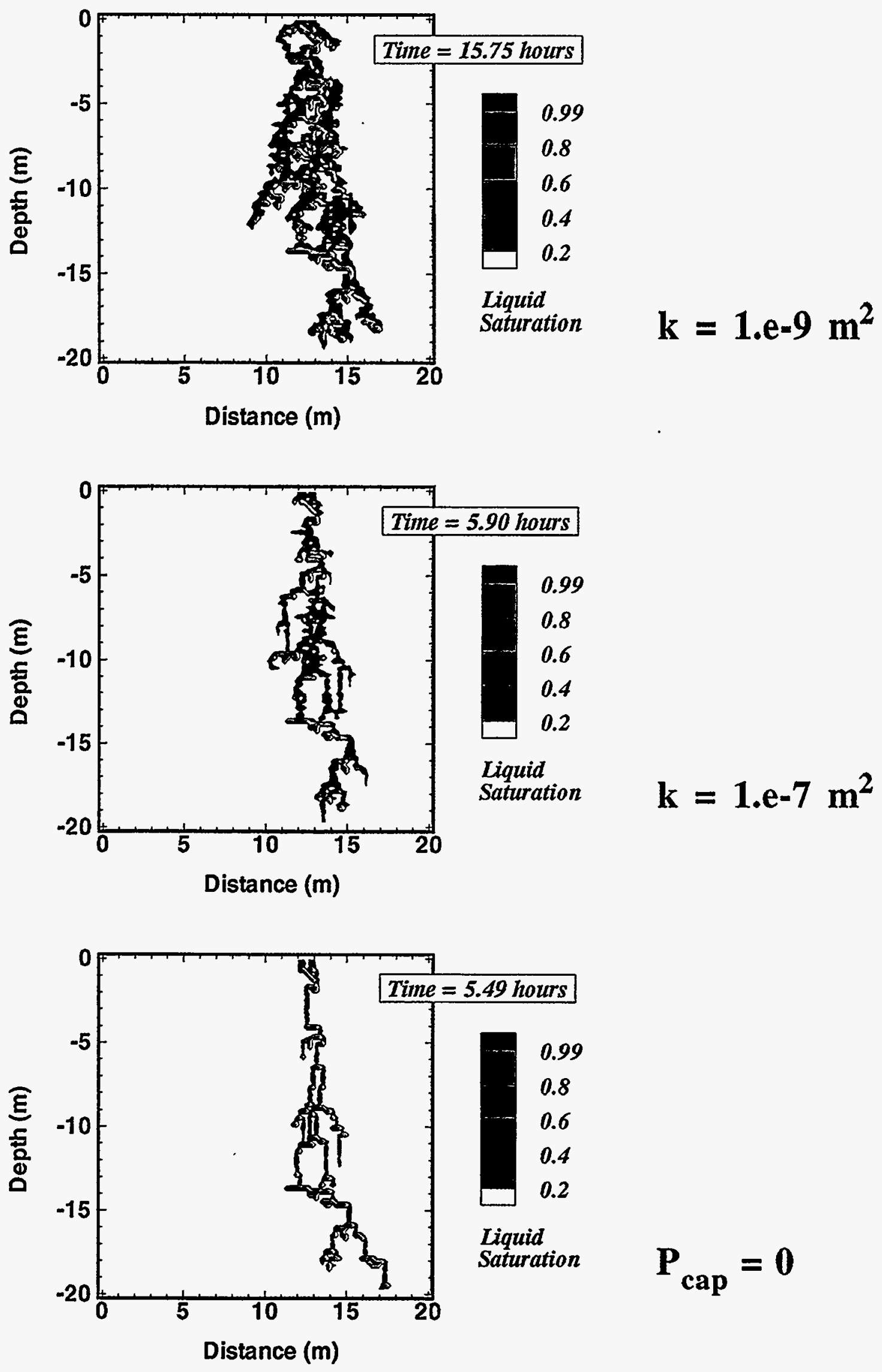

Figure 11. Liquid seeps at breakthrough at $19.5 \mathrm{~m}$ depth for injection at a constant rate of $10^{-3} \mathrm{~kg} / \mathrm{s}$ over a $1 \mathrm{~m}$ interval at the top, centered at $12.5 \mathrm{~m}$. Reference permeability is $10^{-9} \mathrm{~m}^{2}$ (top) and $10^{-7} \mathrm{~m}^{2}$ (middle and bottom). For the seep shown at the bottom, capillary pressure effects were neglected. 

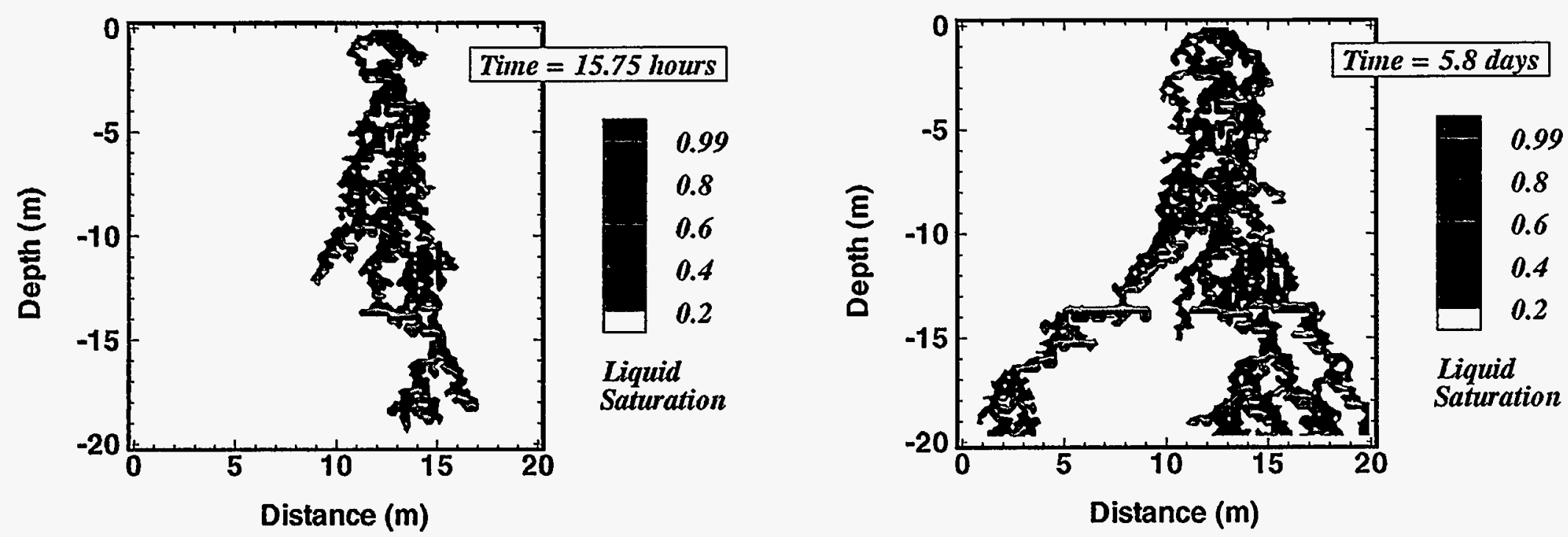

$\stackrel{\omega}{1}$
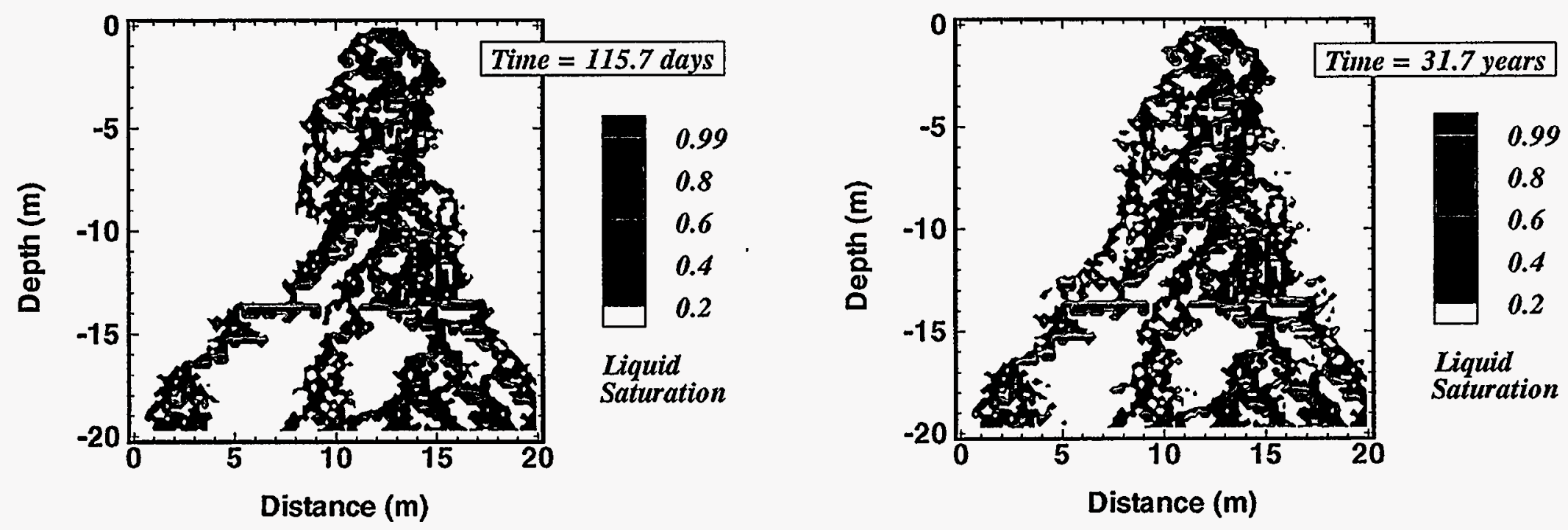

Figure 12. Liquid seeps for injection at a constant rate of $10^{-3} \mathrm{~kg} / \mathrm{s}$ over a $1 \mathrm{~m}$ interval at the top, centered at $12.5 \mathrm{~m}$, shown at four different times. Reference permeability is $10^{-9} \mathrm{~m}^{2}$. 


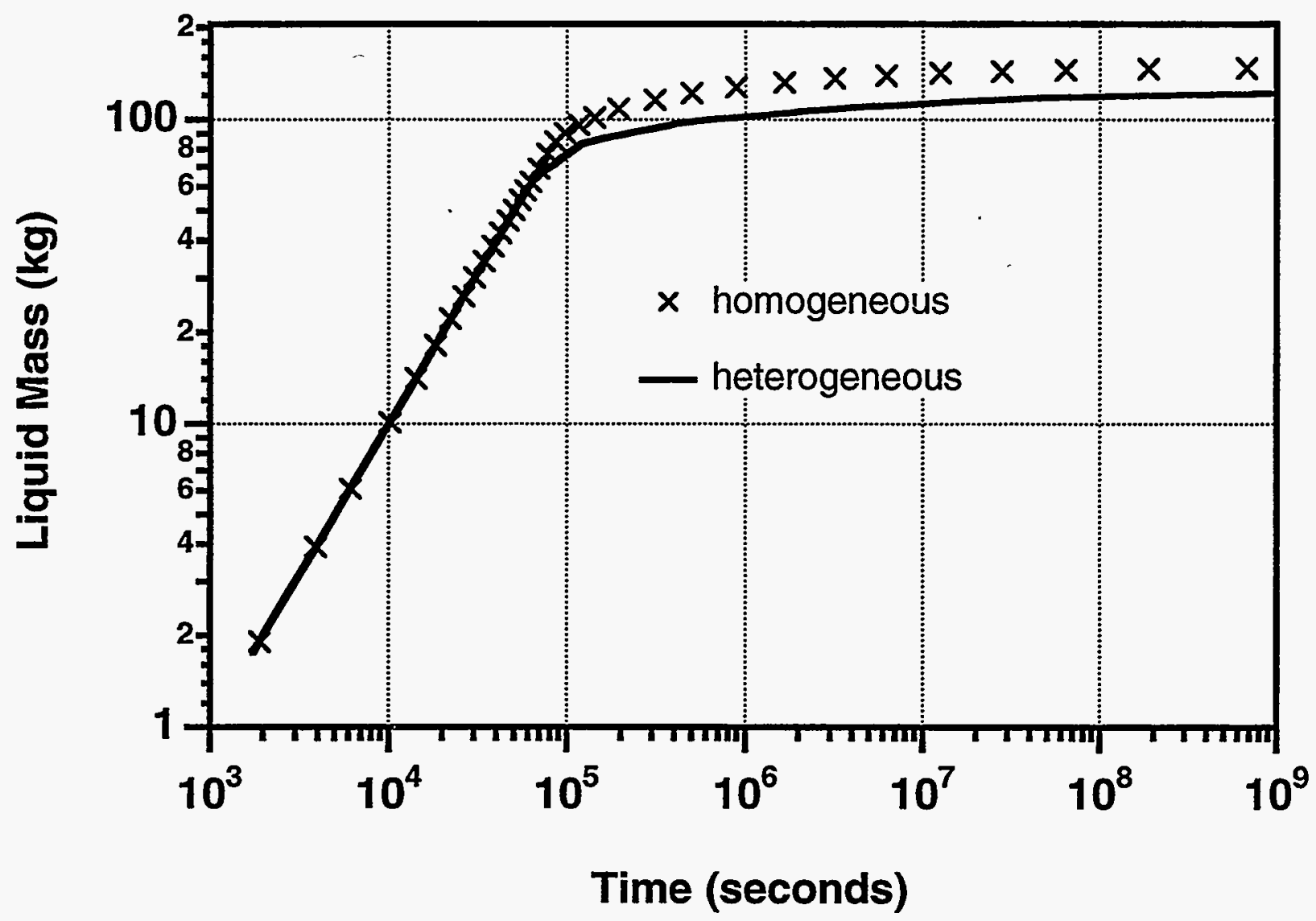

Figure 13. Growth of liquid mass in seep with time for injection at a rate of $10^{-3} \mathrm{~kg} / \mathrm{s}$ at the top boundary of a homogeneous and a heterogeneous fracture. The injection interval is centered at $10.5 \mathrm{~m}$ for the homogeneous and at $12.5 \mathrm{~m}$ for the heterogeneous fracture. 

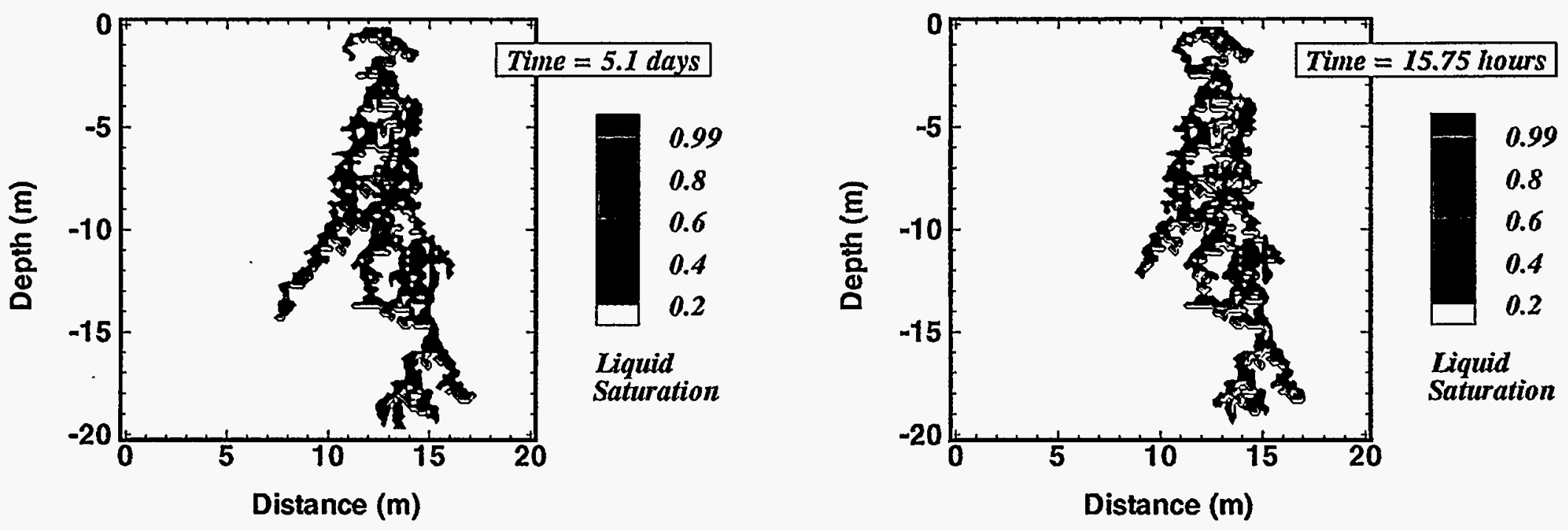

$\omega_{1}^{w}$
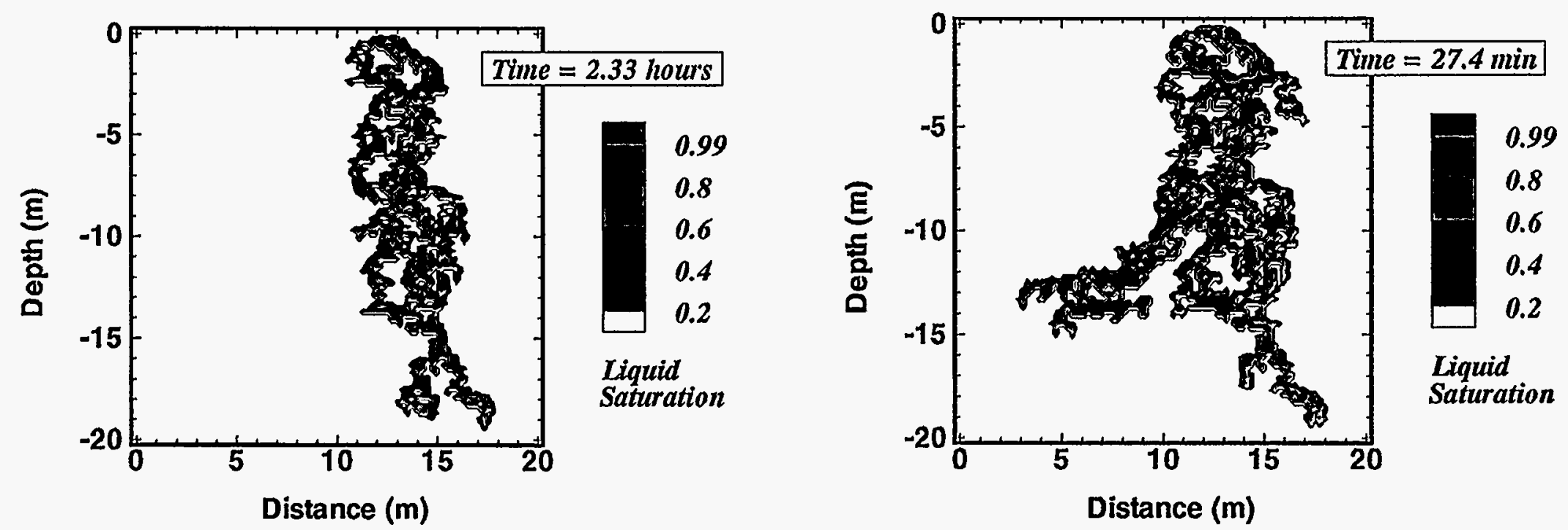

Figure 14. Liquid seeps at time of breakthrough at $19.5 \mathrm{~m}$ depth for injection at four different rates over a $1 \mathrm{~m}$ interval at the top, centered at $12.5 \mathrm{~m}$. Injection rates are $10^{-4} \mathrm{~kg} / \mathrm{s}$ (top left), $10^{-3} \mathrm{~kg} / \mathrm{s}$ (top right), $10^{-2} \mathrm{~kg} / \mathrm{s}$ (bottom left), and $10^{-1} \mathrm{~kg} / \mathrm{s}$ (bottom right). Reference permeability is $10^{-9} \mathrm{~m}^{2}$. 

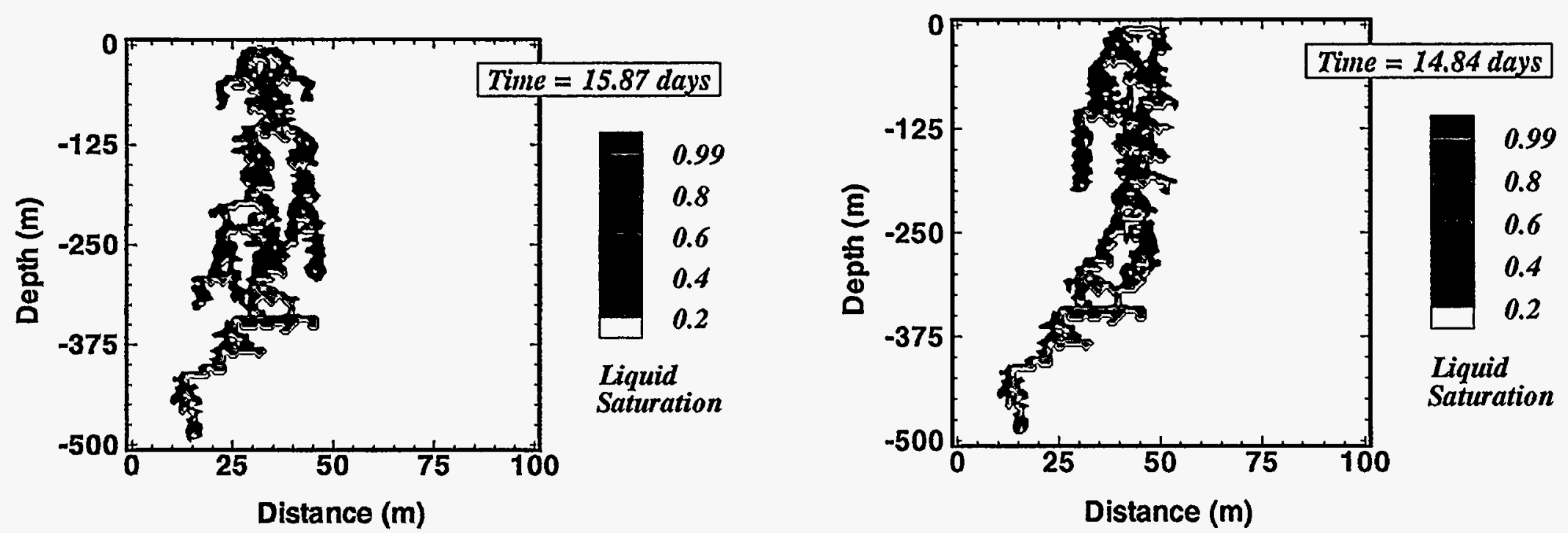

$\stackrel{1}{1}$
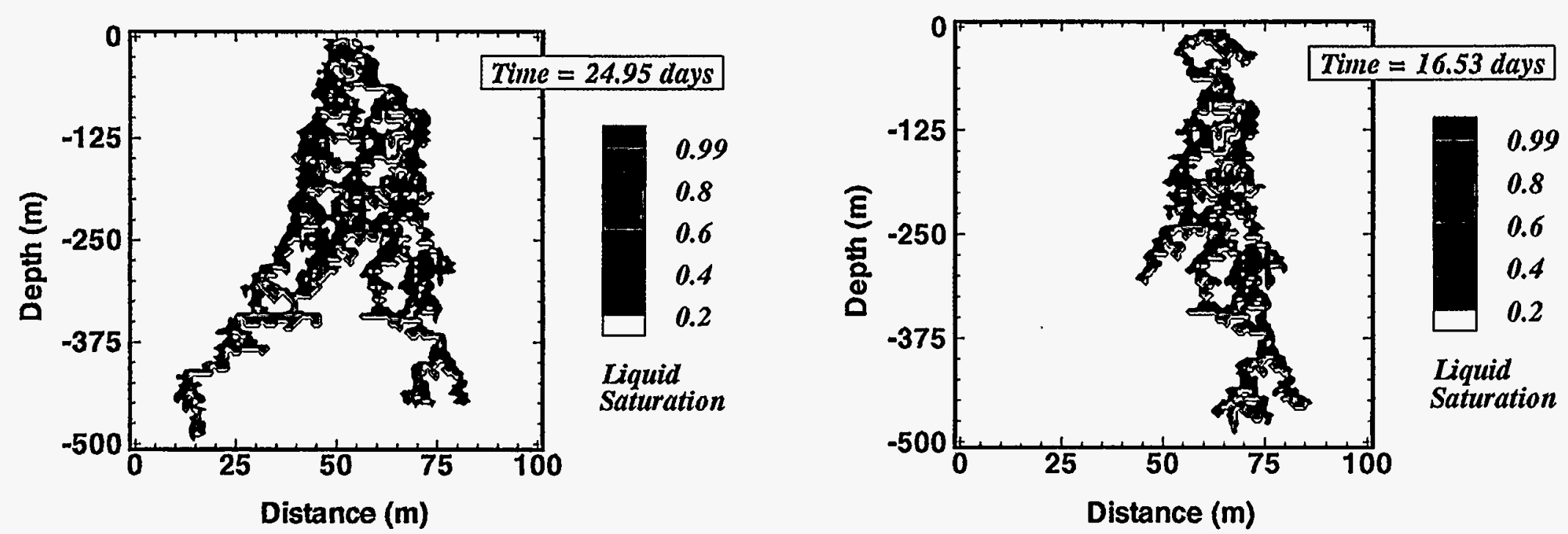

Figure 15. Liquid seeps shown at the time of breakthrough at a depth of $487.5 \mathrm{~m}$. Water is injected at a constant rate of $5 \times 10^{-3} \mathrm{~kg} / \mathrm{s}$ over a $5 \mathrm{~m}$ interval at the top of the fracture. The injection interval is centered at different distances from the left boundary, namely, 32.5 $\mathrm{m}$ (top left), $42.5 \mathrm{~m}$ (top right), $52.5 \mathrm{~m}$ (bottom left), and $62.5 \mathrm{~m}$ (bottom right). Reference permeability is $10^{-9} \mathrm{~m}^{2}$. 

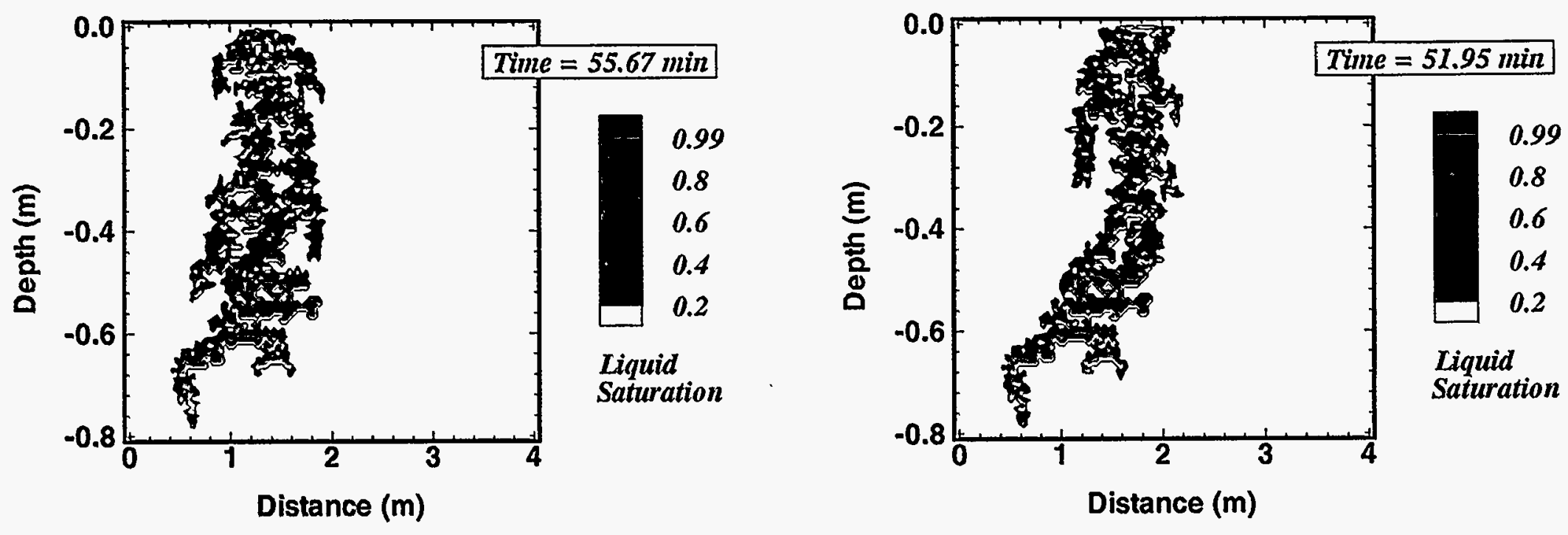

$\ddot{w}_{1}^{\prime}$
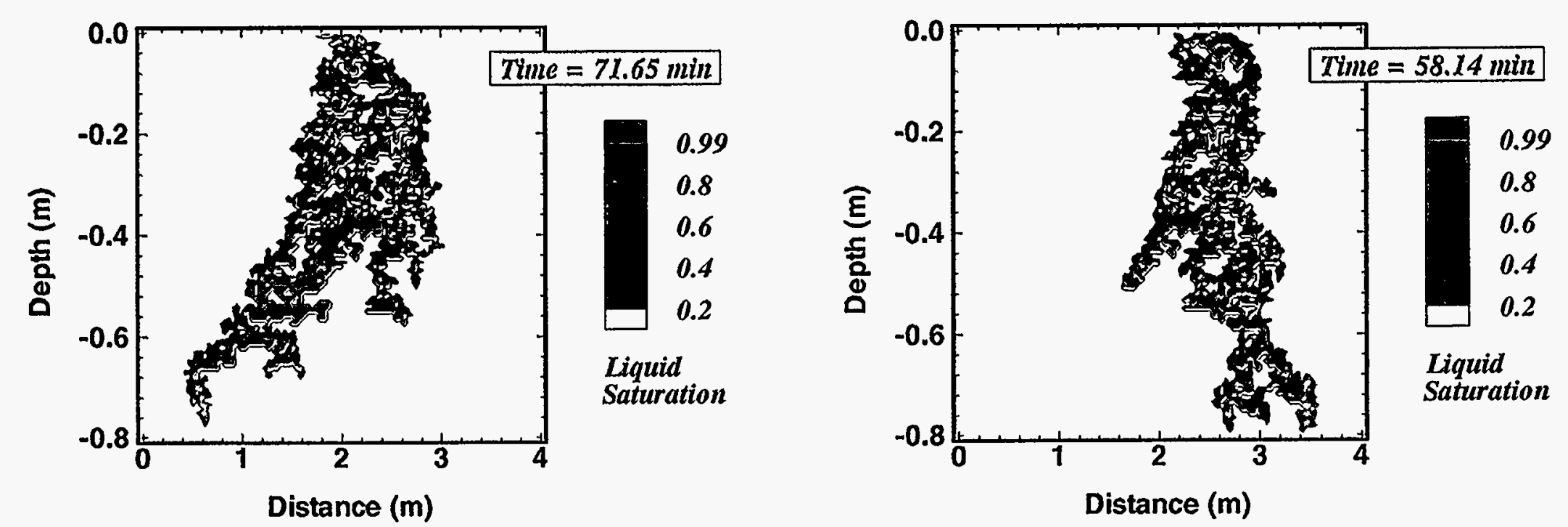

Figure 16. Liquid seeps shown at the time of breakthrough at a depth of $0.78 \mathrm{~m}$. Water is injected at a constant rate of $0.2 \times 10^{-3} \mathrm{~kg} / \mathrm{s}$ over a $0.2 \mathrm{~m}$ interval at the top of the fracture. The injection interval is centered at different distances from the left boundary, namely, $1.3 \mathrm{~m}$ (top left), $1.7 \mathrm{~m}$ (top right), $2.1 \mathrm{~m}$ (bottom left), and $2.5 \mathrm{~m}$ (bottom right). Reference permeability is $10^{-9} \mathrm{~m}^{2}$. 


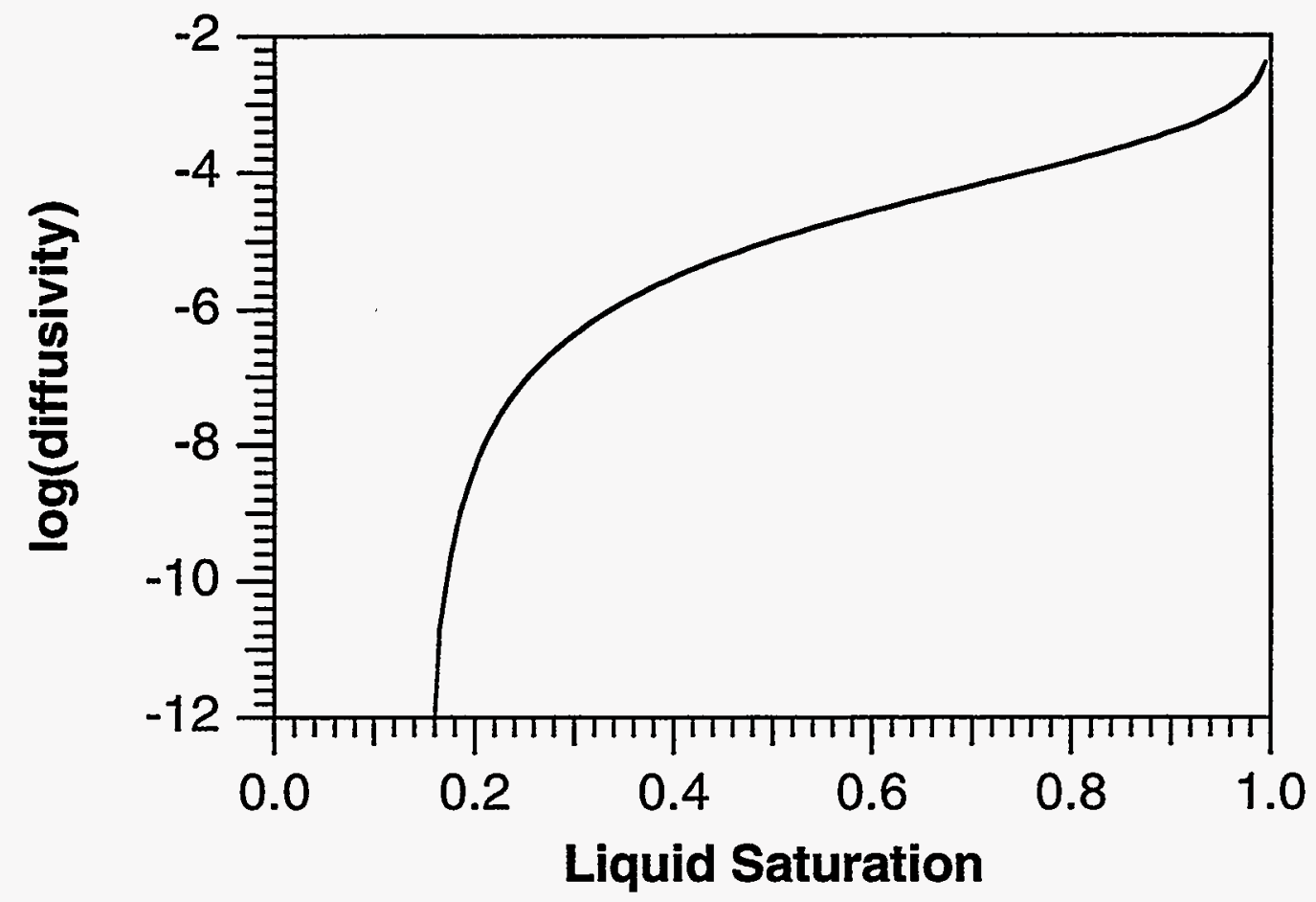

Figure 17. Unsaturated liquid diffusivity for the parameters used in this study (units of $\mathrm{m}^{2} / \mathrm{s}$ ).

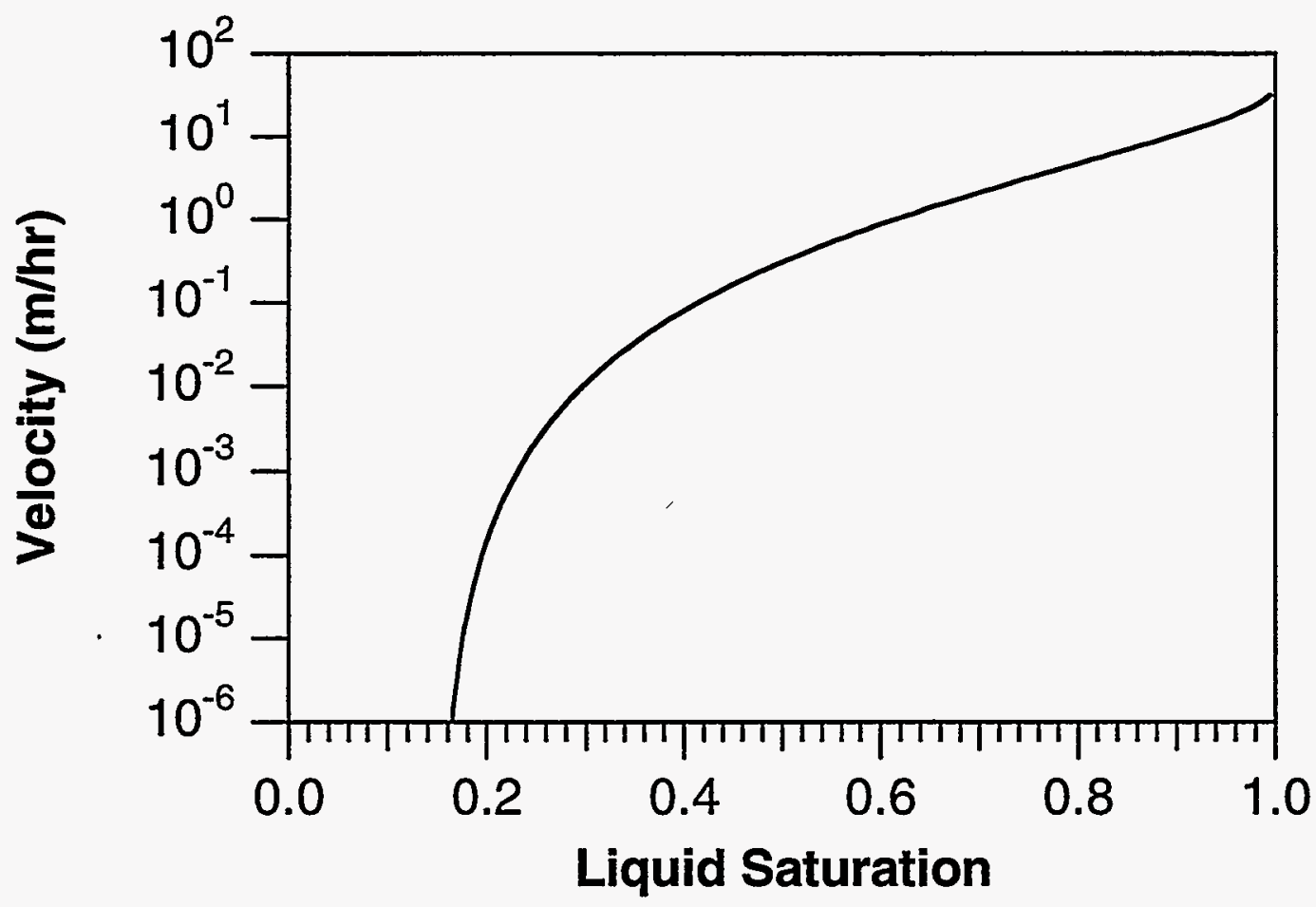

Figure 18. Advective seep velocity for gravity-driven flow in a vertical fracture of permeability $\mathrm{k}_{v}=0.36 \times 10^{-9} \mathrm{~m}^{2}$. 


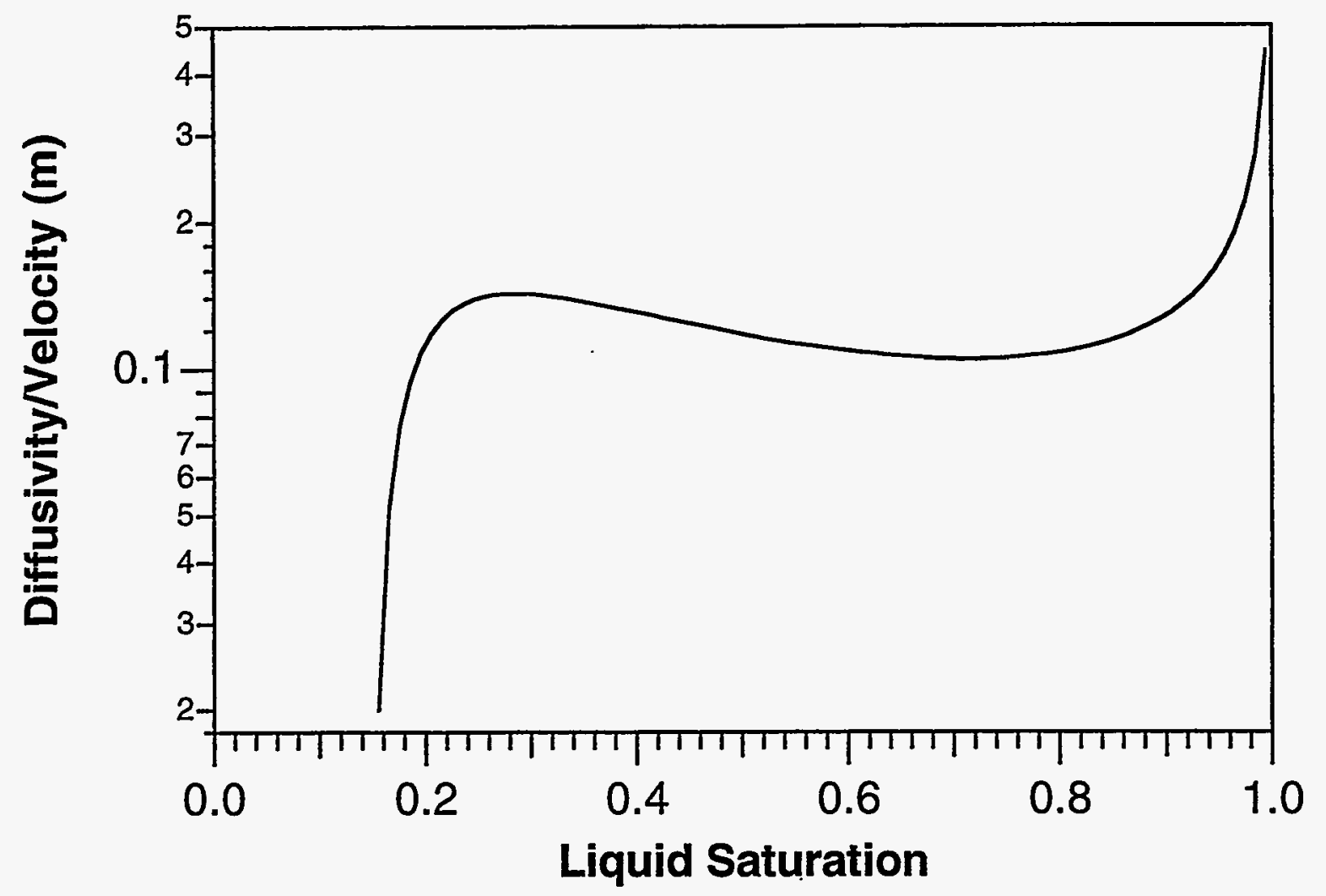

Figure 19. Ratio of capillary diffusivity to advective velocity. 